ARTICLE

\title{
Ybx1 fine-tunes PRC2 activities to control embryonic brain development
}

Myron K. Evans (10 1, Yurika Matsui ${ }^{1,4}$, Beisi Xu (10 ${ }^{2,4}$, Catherine Willis', Jennifer Loome (1) 1, Luis Milburn', Yiping Fan ${ }^{2}$, Vishwajeeth Pagala ${ }^{3} \&$ Jamy C. Peng (iD) ${ }^{1 凶}$

Chromatin modifiers affect spatiotemporal gene expression programs that underlie organismal development. The Polycomb repressive complex 2 (PRC2) is a crucial chromatin modifier in executing neurodevelopmental programs. Here, we find that PRC2 interacts with the nucleic acid-binding protein Ybx1. In the mouse embryo in vivo, Ybx1 is required for forebrain specification and restricting mid-hindbrain growth. In neural progenitor cells (NPCs), Ybx1 controls self-renewal and neuronal differentiation. Mechanistically, Ybx1 highly overlaps PRC2 binding genome-wide, controls PRC2 distribution, and inhibits H3K27me3 levels. These functions are consistent with Ybx1-mediated promotion of genes involved in forebrain specification, cell proliferation, or neuronal differentiation. In Ybx1-knockout NPCs, H3K27me3 reduction by PRC2 enzymatic inhibitor or genetic depletion partially rescues gene expression and NPC functions. Our findings suggest that Ybx1 fine-tunes PRC2 activities to regulate spatiotemporal gene expression in embryonic neural development and uncover a crucial epigenetic mechanism balancing forebrain-hindbrain lineages and selfrenewal-differentiation choices in NPCs.

\footnotetext{
${ }^{1}$ Department of Developmental Neurobiology, St. Jude Children's Research Hospital, 262 Danny Thomas Place, Memphis, TN 38105, USA. ${ }^{2}$ Center for Applied Bioinformatics, St. Jude Children's Research Hospital, 262 Danny Thomas Place, Memphis, TN 38105, USA. ${ }^{3}$ Center for Proteomics and Metabolomics, St. Jude Children's Research Hospital, 262 Danny Thomas Place, Memphis, TN 38105, USA. ${ }^{4}$ These authors contributed equally: Yurika Matsui, Beisi Xu. ${ }^{凶}$ email: jamy.peng@stjude.org
} 
E mbryonic NPCs massively amplify, specify to different brain regions and the spinal cord, and subsequently differentiate into neurons and glial cells. NPC amplification, specification, and differentiation/maturation require an intricate coordination between extracellular signals and cell-intrinsic mechanisms ${ }^{1}$. This coordination is achieved by epigenetic machineries that integrate instructions from signaling pathways and transcription factor networks to output gene expression programs. In developing invertebrate and vertebrate brains, Polycomb group proteins integrate spatial and temporal signals to execute gene expression programs underlying neural diversity ${ }^{2-4}$.

PRC2 is a Polycomb group ribonucleoprotein complex that methylates lysine 27 of histone $\mathrm{H} 3$ (H3K27me) and forms a closed chromatin structure to suppress transcription by RNA polymerase $\mathrm{II}^{5,6}$. In humans, mutations in PRC2 core subunits have been causally linked to Weaver syndrome, a congenital multisystemic syndrome characterized by craniofacial defects, intellectual disabilities, and often macrocephaly, bone and joint malformations, and an increased predisposition to cancer ${ }^{7,8}$. In mice, depletion of the PRC2 core subunits Ezh2, Suz12, and Eed results in gastrulation arrest ${ }^{9-11}$; their knockout $(\mathrm{KO})$ in embryonic stem cells (ESCs) result in failure in neural ectoderm specification $^{12-14}$. Further loss of function studies of PRC2 have demonstrated that PRC2 is required for brain region specification, region-specific proliferation of NPCs, and growth control of brain regions and the spinal cord ${ }^{11,15-17}$. These influences suggest that PRC2 function and regulation vary in different neurodevelopmental contexts.

PRC2 exists as two variant complexes that are defined by their accessory subunits: PRC2.1 associates with subunit EPOP or PALI1 ${ }^{18-20}$, whereas PRC2.2 associates with JARID2 and $A E B P 2^{21}$. These proteins and others likely function distinctively in the PRC2 complex's binding to chromatin, methylating H3K27, and/or suppressing genes. To shed light on PRC2 function and regulation during neural development, we used a proteomics-based approach to identify new interactors of JARID2. JARID2 critically regulates PRC2.2 and neural development: it is required for PRC2.2 to bind chromatin, influences H3K27me levels, and neural tube development. Our effort identified Y-box binding protein $1(\mathrm{Ybx} 1)$ as a previously uncharacterized PRC2-interacting protein.

Ybx1 is a nucleic acid-binding protein known to affect transcriptional activation, DNA repair and replication, RNA processing and stability, and protein translation ${ }^{22,23}$. Through its diverse molecular roles, Ybx1 regulates proliferation, apoptosis, cell differentiation, and cell stress response. For example, Ybx 1 binds the consensus sequence, 5'-CTGATTGG-3', to mediate transcriptional activation of genes involved in epithelial-tomesenchymal transition and drug resistance, likely thereby promoting cancer progression ${ }^{24}$. Its overexpression promotes glioblastoma and medulloblastoma cancer cell proliferation ${ }^{25,26}$. Ybxl depletion results in hematopoiesis failure and exencephaly in the mouse embryo ${ }^{27-29}$. Although Ybx1-KO animals show defects in neural tube closure and growth, the cellular and molecular bases have remained unclear. Here, we show that Ybxl regulates the self-renewal and neuronal differentiation of NPCs in part through regulating PRC2. PRC2 regulation by Ybxl fine-tunes the spatiotemporal expression of neurodevelopmental genes.

\section{Results}

YBX1 is a candidate PRC2-interacting factor. We used mass spectrometry to detect endogenous proteins that coimmunoprecipitated with JARID2 from the human ESC (hESC) nuclear extract. We used a validated, specific anti-JARID2 antibody (Supplementary Fig. S1a, b), which did not coimmunoprecipitate G9A in the hESC nuclear extract (Supplementary Fig. S1c). In addition to JARID2, we detected the known PRC2 subunits EZH2, SUZ12, EED, AEBP2, and RBBP4 (Supplementary Fig. S1d, e). Importantly, we detected YBX1 (Supplementary Fig. S1d, e), which was also uncovered by Ezh2 immunoprecipitation-mass spectrometry from NT2 cells ${ }^{30}$. These provided the rationale for our study to examine Ybxl's function and interaction with PRC2.

Nuclear Ybx1 modulates the self-renewal of NPCs. Ybx1-KO mice are known to display a strong, highly penetrant exencephaly phenotype ${ }^{27,29}$. We analyzed $Y b \times 1-\mathrm{KO}$ mice in a back-crossed C57BL/6 background and found that $\sim 84 \%$ displayed exencephaly, confirming previous findings (Supplementary Fig. S2a, b). Exencephaly can be caused by deregulation of NPCs, so we examined how Ybxl affects NPC properties. First, we examined NPC proliferation in $\mathrm{Ybx1-KO}$ embryos and sibling controls by immunofluorescence (IF) and fluorescence-activated cell sorting (FACS). To identify proliferating cells in vivo, we injected BrdU into pregnant dams and/or detected the mitotic chromatin marker phosphorylated-serine 10 in histone $\mathrm{H} 3$ (PH3; Fig. 1a). Mouse NPCs were identified by their expression of the neural stem cell marker Sox2. IF of cryosections of embryonic day 13.5 (E13.5) mouse neural tubes showed a significant increase in the number and frequency of proliferating BrdU-positive NPCs in Ybx1-KO embryos compared to controls $(P<0.0001$; Fig. $1 \mathrm{~b}, \mathrm{c})$. As the number of Sox2-positive NPCs in Ybx1-KO embryos was significantly higher than that in control embryos, we normalized BrdU counts to the total number of Sox2-positive cells that remained significant when compared to the control $(P<0.05$; Fig. 1d).

FACS analysis showed that, on average, the proportion of BrdU-positive NPCs was $\sim 14 \%$ in control and $\sim 26 \%$ in $Y b \times 1-\mathrm{KO}$ neural tubes at E13.5, which confirmed the IF results (Supplementary Fig. S2c, d). Quantification of matched littermate embryos revealed that there were significantly more BrdUpositive $(P<0.01$; Supplementary Fig. S2d) and $\mathrm{PH} 3$-positive $(P<0.05$; Supplementary Fig. S2d) NPCs in Ybx1-KO than in sibling control embryos. In contrast, $Y b \times 1-\mathrm{KO}$ mouse embryonic fibroblasts (MEFs) displayed decreased proliferation compared to controls (Supplementary Fig. S2e-g), consistent with previous reports ${ }^{27}$. These data suggest that Ybx1 has different cellular influences in different developmental contexts: promoting the proliferation of fibroblasts and suppressing the proliferation of NPCs.

We used the neurosphere assay to examine the effect of $\mathrm{Ybx} 1$ over self-renewal. Mouse NPCs were purified by Neurofluor ${ }^{\mathrm{m}}$ CDr3 FACS (which targets NPC marker FABP7 ${ }^{31}$ ) and allowed to form neurospheres that were serially passaged (Fig. 1e). We quantified neurosphere number and area and found that, by the third passage, both were higher for Ybx1-KO compared to controls (Fig. 1f-h). A separate clonal assay confirmed that Ybx1KO NPCs had increased self-renewal compared to controls (Supplementary Fig. S2i, j).

To investigate the nuclear role of Ybx1 in NPC proliferation, we transduced $Y b x 1-\mathrm{KO}$ NPCs with viruses expressing wild-type YBX1 or a mutant without the nuclear localization signal (delNLS from deletion of amino acids 183-205; Fig. 1i). In transduced Ybx1-KO NPCs, wild-type YBX1 proteins were expressed in both the nucleus and cytoplasm, whereas the delNLS-YBX1 proteins were expressed only in the cytoplasm (Fig. $1 \mathrm{j}$ and Supplementary Fig. S2k). While expression of PRC2 subunits were unaffected, wild-type (but not delNLS) YBX1 restored the expression of some forebrain lineage genes (Supplementary Fig. S2l). We found that 
a
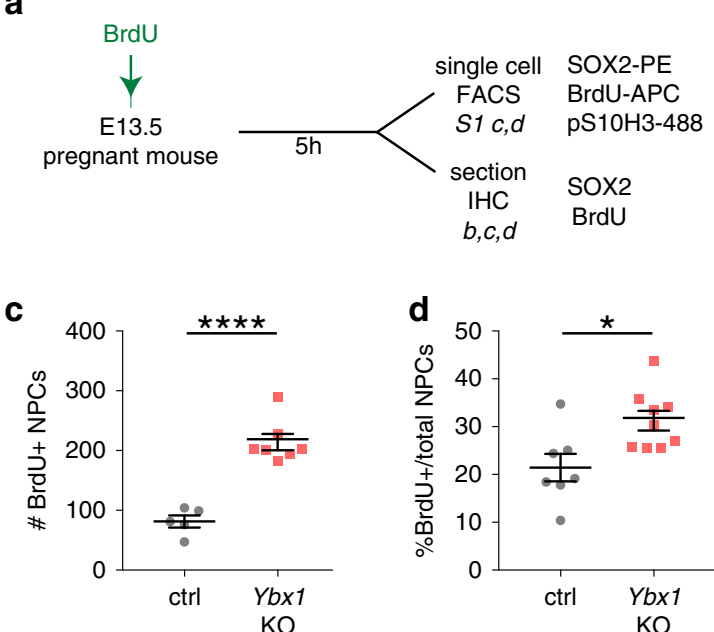

$\mathbf{e}$
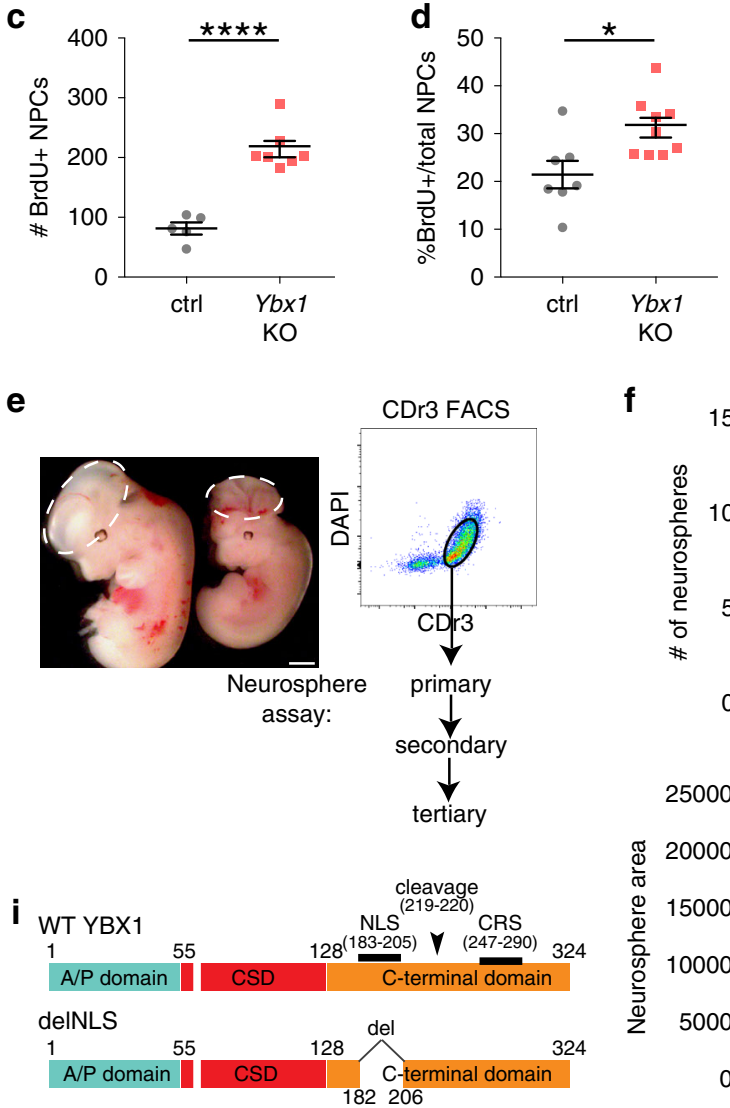

KO b

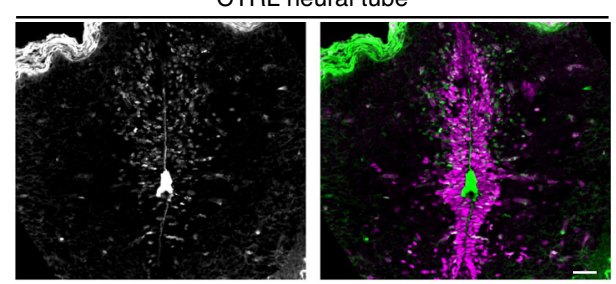

Ybx1-KO neural tube

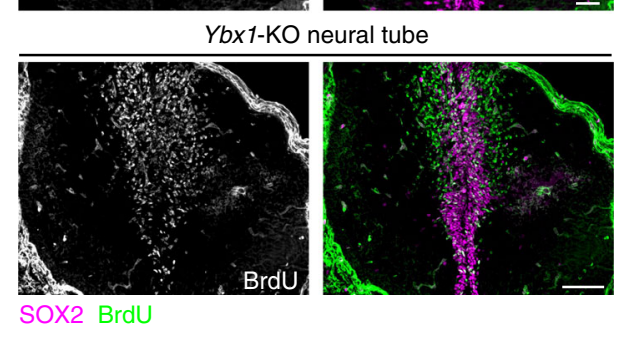

SOX2 BrdU
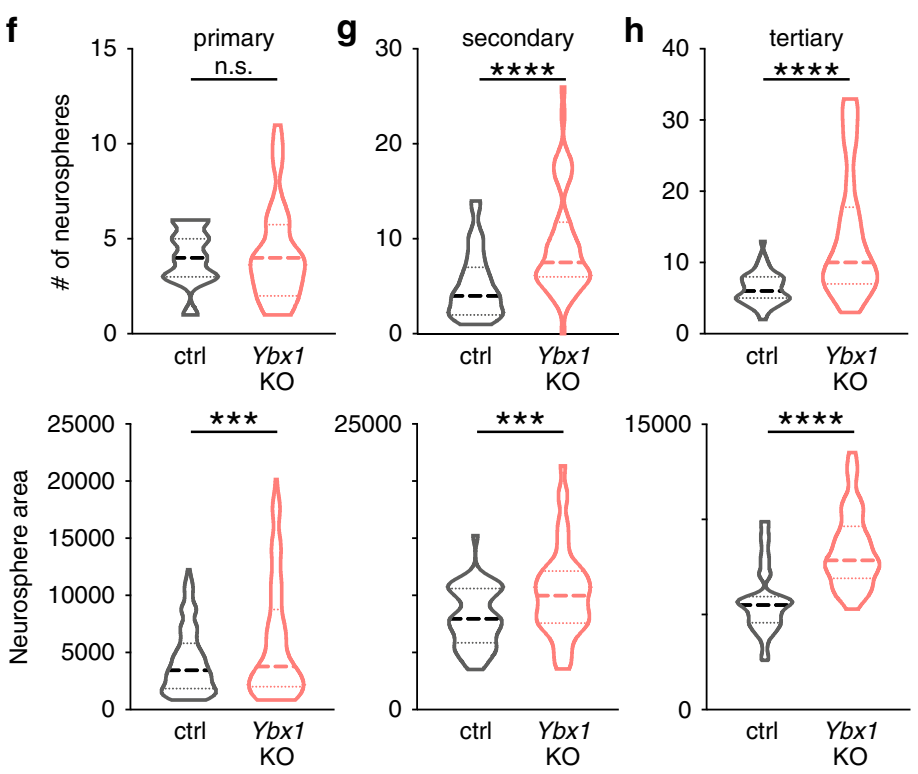

j
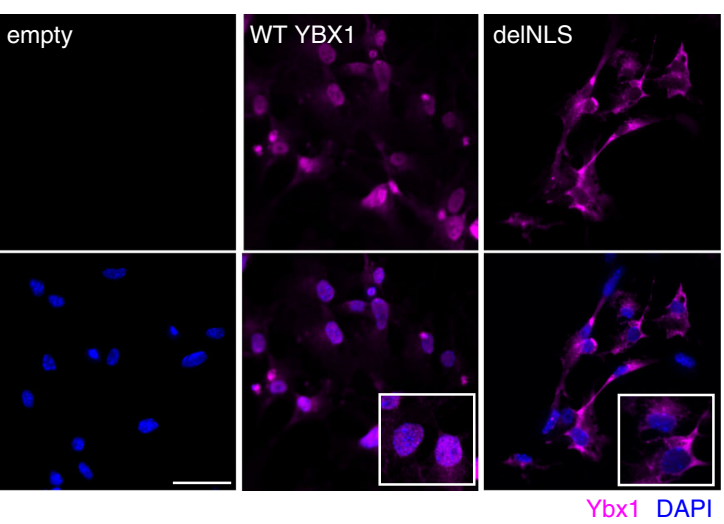

$\mathbf{k}$
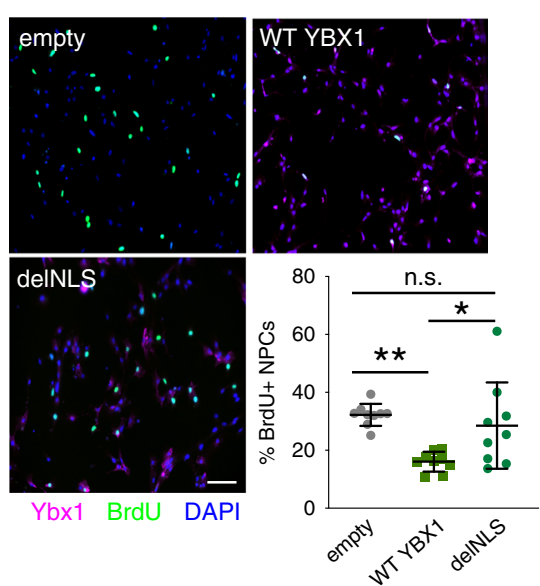

wild-type YBX1 resulted in reduced proliferation of Ybx1-KO NPCs, whereas delNLS-YBX1 did not significantly affect the proliferation of Ybx1-KO NPCs (Fig. 1k). These data suggest that nuclear Ybx1 modulates the self-renewal of NPCs.

Ybxl is required for neurodevelopmental gene expression. To examine how Ybxl affects gene expression in NPCs, we performed RNA-sequencing (RNA-seq) of Sox2-positive NPCs sorted from E13.5 Ybx1-KO and sibling controls (Supplementary Fig. S3a). We analyzed expressed genes as those having FPKM values $>1$ in either control or Ybx1-KO NPCs. Using the criteria of adjusted $P<0.05$ and fold-change $>1.5$ to compare three datasets each from control and Ybx1-KO NPCs, we identified 604 upregulated genes and 366 downregulated genes in Ybx1-KO 
Fig. 1 Ybx1-KO NPCs had increased self-renewal. a Schematic for BrdU labeling and analyses of mouse embryos. b IF of Sox2 and BrdU in cryosections from Ybx1-KO or sibling control embryos at E13.5. Bar, $100 \mu \mathrm{m}$. Quantification of IF in cryosections to determine the $\mathbf{c}$ number of BrdU-positive NPCs per cryosection ( $n=7$ /genotype) or d percentage of BrdU-positive NPCs ( $n=7$ control or 9 Ybx1-KO biologically independent embryos) from Ybx1-KO and sibling control embryos. e Diagram of NPC isolation by CDr3 FACS and subsequent neurosphere formation and serial passages. Bar, $10 \mu \mathrm{m}$. Quantification of the number and area of $\mathbf{f}$ primary, $\mathbf{g}$ secondary, and $\mathbf{h}$ tertiary neurospheres formed by NPCs from Ybx1-KO and sibling control embryos. $\mathbf{i}$ Diagrams of WT full-length (FL) and mutant deINLS-YBX1 cDNAs. $\mathbf{j}$ Ybx1 IF in Ybx1-KO NPCs transduced with lentiviruses from empty vector, WT YBX1, or

deINLS-YBX1. Inserts contain zoom-in images of representative cells. Bar, $50 \mu \mathrm{m}$. $\mathbf{k}$ YBX1 and BrdU IF in Ybx1-KO NPCs transduced with lentiviruses from empty vector, WT YBX1, or deINLS-YBX1. $n=9$ images from one experiment, which was repeated twice. Bar, $50 \mu \mathrm{m}$. Quantification of NPCs transduced with different lentiviruses. Data are presented as mean \pm SEM for $\mathbf{c}$, $\mathbf{d}$, and $\mathbf{k}$ and as violin plot of frequency distribution (lines at median and quartiles) in $\mathbf{f}-$ h. $P$ values by two-tailed unpaired $t$ test are indicated. n.s. indicate not significant. ${ }^{\star}$, ${ }^{\star \star}$, ${ }^{\star \star \star}$, and ${ }^{\star \star \star \star}$ indicate $P<0.05,0.01,0.001$, and 0.0001, respectively. Source data are provided in Source Data file. $P$ values: $1 \mathbf{c}-<0.0001 ; 1 \mathbf{d}-0.01 ; 1 \mathbf{f}$ (bottom)-0.0005; $1 \mathbf{g}$ (top)-<0.0001; $1 \mathbf{g}$ (bottom)-0.002; $1 \mathbf{h}$ (top)-<0.0001, 1h (bottom)-<0.0001; 1k(empty-WT)-0.003, 1k(WT-delNLS)-0.02.

(Fig. 2a, Supplementary Fig. S3b). Many of the differentially expressed genes were involved in nervous system development or cell fate (Fig. 2b). Gene ontology and gene set enrichment analysis (GSEA) revealed that downregulated genes in Ybx1-KO NPCs were enriched in functions related to central nervous system, neuronal differentiation, and morphogenesis (Fig. 2c, d). Upregulated genes in Ybx1-KO NPCs were enriched in functions related to embryo development, transcriptional regulation, cell proliferation, and known YBX1 targets ${ }^{32}$ (Fig. 2e, f). These data suggest that Ybxl suppresses genes involved in cell proliferation and promotes genes involved in neuronal differentiation.

As many differentially expressed genes in Ybx1-KO NPCs were related to brain development, we wanted to determine whether the observed changes in gene expression occurred in NPCs from the brain, the spinal cord, or both. Thus, we separately purified NPCs (by Neurofluor ${ }^{\mathrm{m}}$ CDr3 FACS) from these 2 regions and performed RT-qPCR. Neurodevelopmental genes that we analyzed were significantly downregulated in brain NPCs but not similarly affected in spinal cord NPCs (Supplementary Fig. S3c). Therefore, we focused on Ybxl in the developing brain.

Our RNA-seq analysis showed that several crucial forebrain lineage genes were downregulated in Ybx1-KO NPCs. These genes included $F g f 8^{33}, \operatorname{Six} 3^{34,35}, E m \times 2^{36}, \operatorname{Ar} x^{37}$, and $D k k 1^{38}$, which are required for the patterning and formation of the forebrain. Other downregulated genes included Hes5, which is required for NPC proliferation and differentiation to neurons ${ }^{39,40}$, and Fezf2, which is required for neuronal differentiation in the forebrain ${ }^{41}$. TaqMan ${ }^{\text {TM }}$ assays confirmed the downregulation of forebrain lineage genes (Fig. $2 \mathrm{~g}$ ) and the upregulation of midbrain and hindbrain lineage genes (Supplementary Fig. S3d) in Ybx1-KO NPCs. IF analysis of E13.5 sagittal cryosections confirmed the decreased expression and coverage of the forebrain marker Foxg1 (Fig. 2h), whereas midbrain and hindbrain regions positive for the marker Gbx2 were expanded (Supplementary Fig. S3e) in Ybx1-KO compared to controls. We infer that reduced forebrain specification in Ybx1-KO embryos led to the overgrowth of the midbrain and hindbrain. We concluded that Ybxl regulates the expression of genes that are crucial for brain patterning and formation, NPC proliferation, and neuronal differentiation.

Ybxl is required for the differentiation of NPCs to neurons. Our RNA-seq results suggest that in NPCs, Ybxl is required to promote the expression of neuronal differentiation genes (Fig. $2 \mathrm{c}$, d). Therefore, we used a 14-day in vitro differentiation assay (Fig. 3a) to test whether Ybx1 affects the differentiation of NPCs (purified by Neurofluor ${ }^{\mathrm{Ts}}$ CDr3 FACS) to neurons (Tuj1 marker) or glia (Gfap marker). This assay enabled us to focus on the cellintrinsic role of Ybx1 in NPCs. We observed reduced expression of neuronal markers in differentiating cells from $Y b x 1$-KO versus control (Fig. 3b, c). Quantification of IF showed significantly fewer Tuj1-positive neurons and shorter and less-arborized neurites in the sparse Tuj1-positive neurons of Ybx1-KO than in sibling controls (Fig. $3 \mathrm{~d}-\mathrm{g}$ ). Although we detected a modest and significant increase in glial gene expression in Ybx1-KO differentiating cells, the number of Gfap-positive cells were not significantly different between Ybx1-KO and control (Fig. 3h). These findings are consistent with a previous report that sustained Ybx1 expression correlates with neuronal differentiation whereas Ybx1 loss correlates with gliogenesis ${ }^{25}$. We conclude that Ybx1 is required for an NPC-intrinsic mechanism to promote gene expression during neuronal differentiation.

Ybx1 regulates the expression of neurodevelopmental genes. We profiled the genome-wide distribution of Ybxl by CUT\&RUN-seq ${ }^{42}$ in $Y b x 1-\mathrm{KO}$ and sibling control NPCs (by Sox2-GFP FACS; Fig. 4a). ChIP-seq and CUT\&RUN-seq were performed with spike-in method: Drosophila S2 chromatin for histone profiling and H2Av CUT\&RUN of S2 cells for Ybx1 CUT\&RUN-seq. The heat map in Fig. 4a shows Ybx1 distribution in Ybx1-KO and control NPCs. Using a $10^{-3}$ false discovery rate to analyze seq read counts per million, we identified 23,070 Ybx1bound regions in NPCs and only 493 (445 overlapping those in control) regions in Ybx1-KO NPCs; showing the high specificity of Ybx1 CUT\&RUN-seq. Approximately 46\% of Ybx1-bound peaks were within $10 \mathrm{bp}$ of the transcription start site (TSS; Supplementary Fig. S4a) and $58.8 \%$ were within promoters (within $2 \mathrm{~kb}$ of transcription start sites; Supplementary Fig. S4b). Of Ybxl-bound peaks, most located in protein coding genes (Supplementary Fig. S4b). Only $15.3 \%$ of Ybx1-bound peaks were located distal to a gene.

We found that 382 of the 604 (63\%) upregulated genes and 263 of the $366(68 \%)$ downregulated genes in Ybx1-KO NPCs were bound by Ybx1 in NPCs (Fig. 4b). The increased frequency of Ybxl binding in downregulated genes versus upregulated genes was significant $(P=0.0062$ by two-tailed Fisher's exact test; Fig. $4 \mathrm{~b})$. The downregulated Ybx1-bound genes were enriched in functions related to nervous system development, neuron differentiation, synapse transmission, axon guidance, glutamate signaling, and BDNF signaling (Supplementary Fig. S4c), suggesting that Ybx1 directly promotes the expression of genes related to brain development, neurogenesis, and neuronal function. Upregulated Ybxl-bound genes were enriched in functions related to neural crest differentiation, transcriptional regulation, cell cycle, dopaminergic neurogenesis, transcriptional misregulation in cancer, and Hox genes in hindbrain development (Supplementary Fig. S4d), suggesting that Ybx1 directly suppresses genes involved in these functional categories. Unsupervised clustering of differentially expressed genes in these categories effectively separated control and Ybx1-KO NPCs (Supplementary Fig. S4e), further supporting that $\mathrm{Ybx} 1$ directly regulates the expression of neurodevelopmentally important genes. 
a

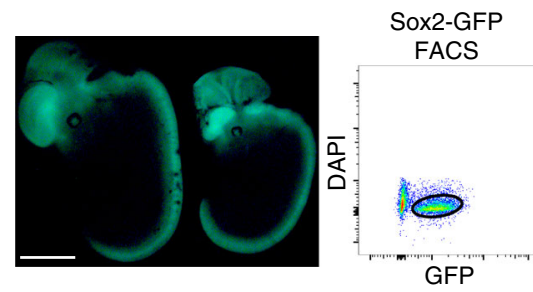

Differentially expressed genes $>1.5$ fold

upregulated downregulated

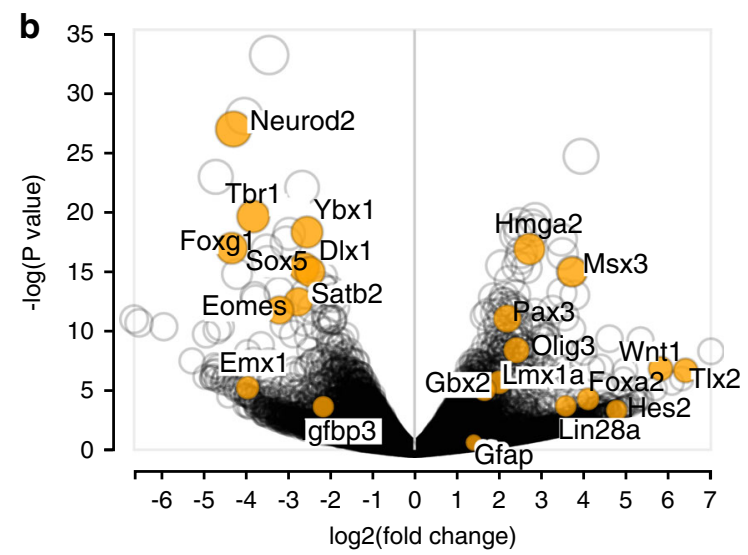

C

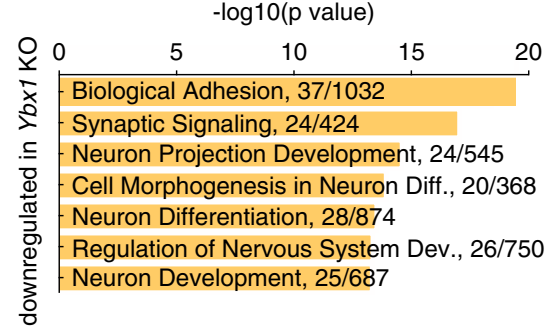

e

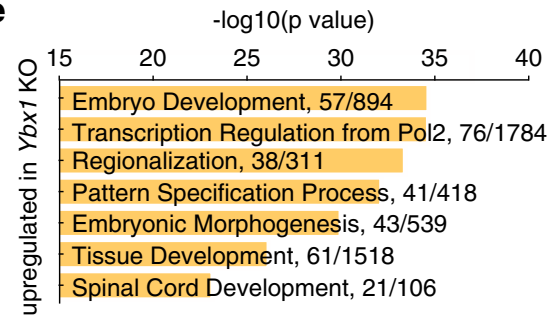

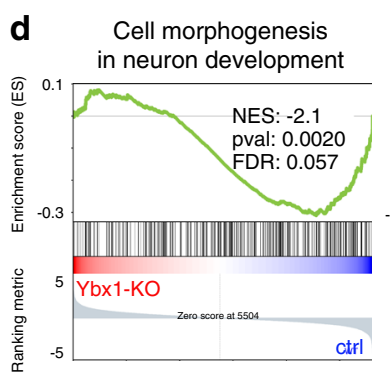
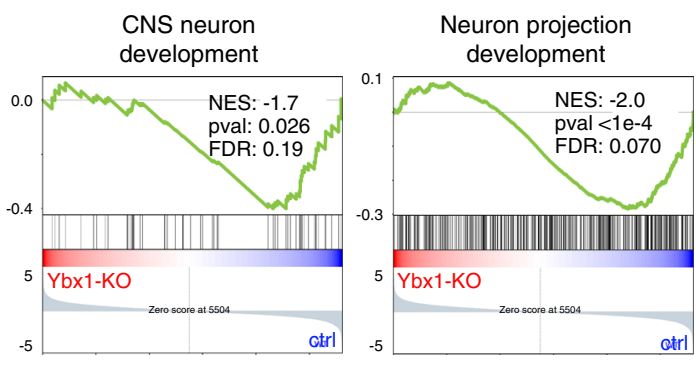

f
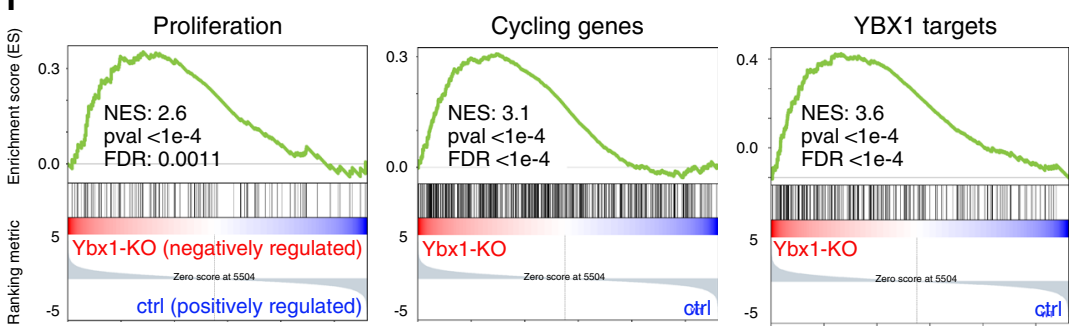

ctrl g

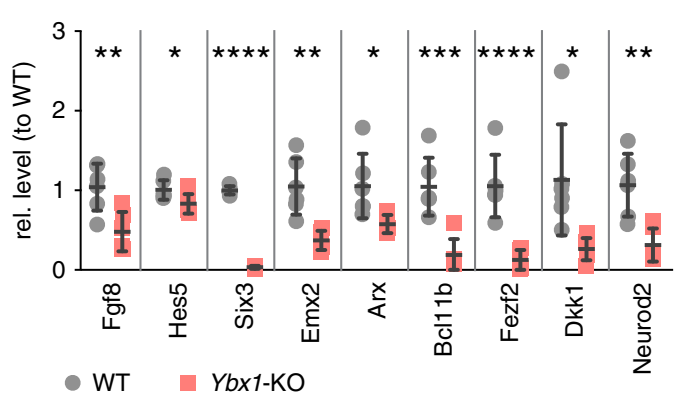

h

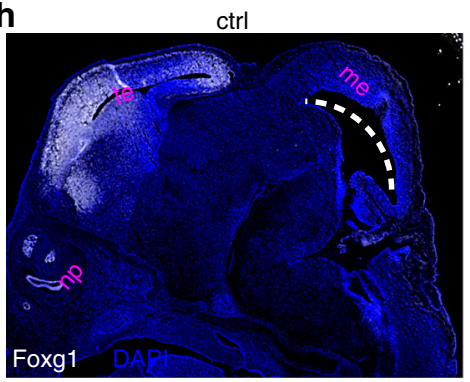

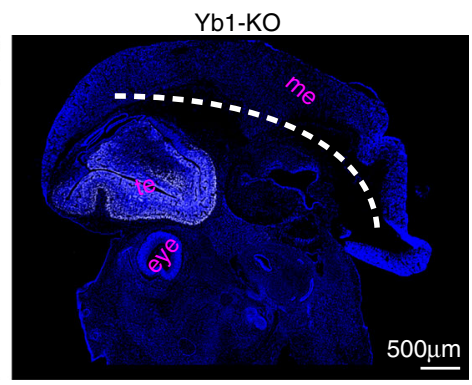

Fig. 2 Differential gene expression in control and Ybx1-KO NPCs purified from embryos. a Image of Ybx1-KO and sibling control embryos expressing Sox2-GFP. Bar, 2 mm. Representative plot of Sox2-GFP FACS. Summary of differentially expressed genes in Sox2-GFP-positive NPCs from Ybx1-KO. b Volcano plot comparing transcript profiles of four samples of control NPCs and three samples of Ybx1-KO NPCs. $p$-values by two-tailed unpaired $t$ test in limma package. Gene ontology analysis identified ontology terms of $\mathbf{c}$ downregulated or e upregulated genes in Ybx1-KO vs. control NPCs. Terms were ranked by $P$ value, calculated by two-tailed Fisher's exact test, with the number of enriched genes indicated. GSEA identified enrichment gene sets in d downregulated or $\mathbf{f}$ upregulated genes in Ybx1-KO NPCs. $P$ values were calculated by one-tailed Kolmogorov-Smirnov statistic test. $\mathbf{g}$ RT-qPCR with TaqMan assays of forebrain lineage markers in NPCs purified from brains of Ybx1-KO or sibling control embryos at E13.5. $n=6$. Data are presented as mean \pm SEM for $\mathbf{g}$. $P$ values by two-tailed unpaired $t$ test are indicated. n.s., not significant. ${ }^{\star},{ }^{\star \star},{ }^{\star \star \star}$, and ${ }^{\star \star \star \star}$ indicate $P<0.05,0.01,0.001$, and 0.0001 , respectively, by one-sided Student's $t$ test. h Foxg1 IF in cryosection from Ybx1-KO or sibling control embryos at E13.5. Bar, 500 $\mu \mathrm{m}$. Te telencephalon, me mesencephalon, np nasal plate. Source data are provided in Source Data file. P values: $2 \mathbf{g}$ (Fgf8)-0.005, (Hes5)-0.03, (Six3)-<0.0001, (Emx2)-0.001, (Arx)-0.03, (Bcl11b)-0.0005, (Fezf2)-0.0003, (Dkk1)-0.01 (NeuroD2)-0.002. 
a

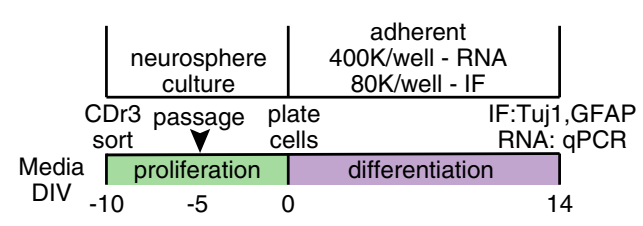

b

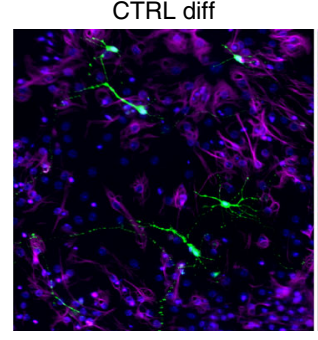

Ybx1-KO diff

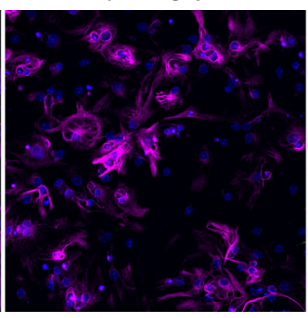

Ybx1-KO diff

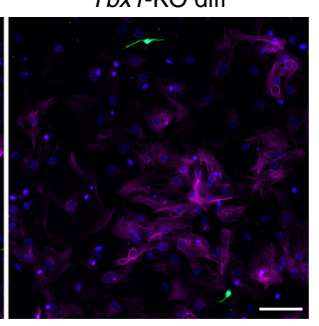

GFAP Tuj1 DAPI
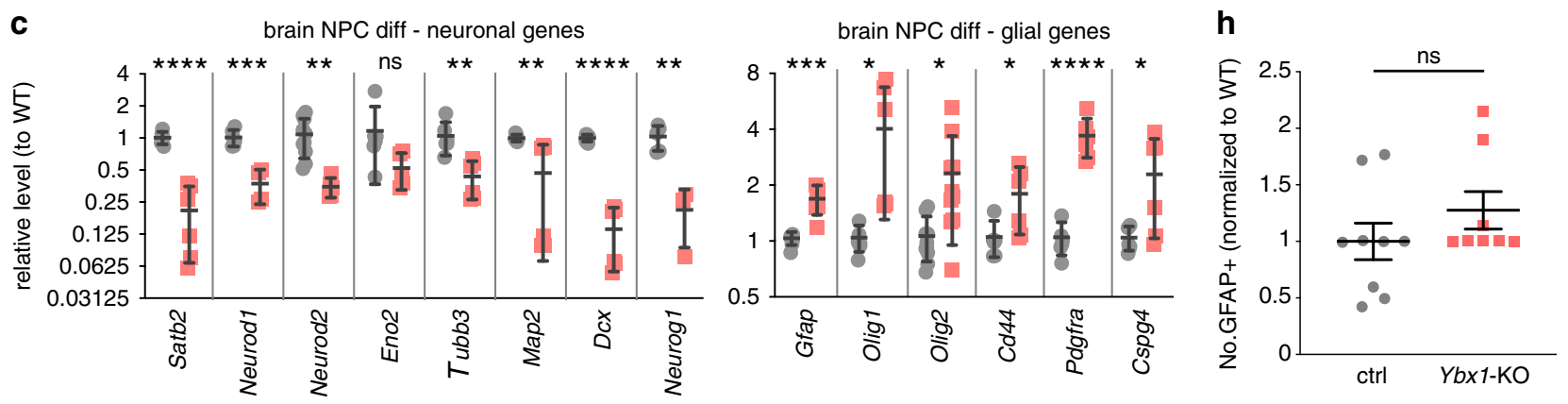

- WT $\square \mathrm{Ybx1 \textrm {KO }}$
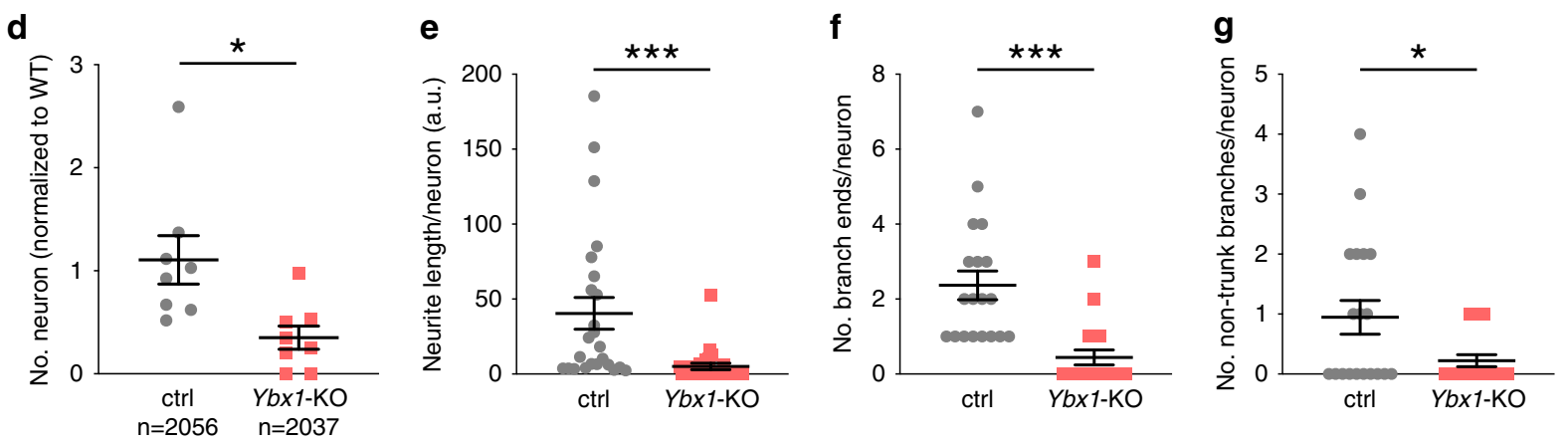

Fig. 3 Ybx1 is required for the differentiation of NPCs to neurons. a Diagram illustrating the workflow of in vitro differentiation of NPCs. $\mathbf{b}$ IF of Tuj1 and Gfap in Ybx1-KO and sibling control differentiating cells. c RT-qPCR with TaqMan assays of neuronal and glial genes in control and Ybx1-KO differentiating cells at day 14. $n=6$ /genotype. $n=10$ for Neurod2 in WT, and $n=10$ for Olig2 (see source data). Quantification of $\mathbf{d}$ relative number of Tuj1-positive neurons, e neurite length, $\mathbf{f}$ number of branch points in axons, $\mathbf{g}$ number of non-trunk branches (not connected to axons or dendrites; $n=8$ images and cell numbers indicated), and $\mathbf{h}$ number of Gfap-positive (glial marker; $n=9$ control images and 8 Ybx 1 -KO images) cells in Ybx $1-K O$ and sibling control differentiating cells. Data are presented as mean \pm SEM for $\mathbf{c}-\mathbf{h}$. $P$ values by two-tailed unpaired $t$ test are indicated. n.s. indicates not significant. ${ }^{*},{ }^{\star \star},{ }^{\star \star \star}$, and ${ }^{\star \star \star \star}$ indicate $P<0.05,0.01,0.001$, and 0.0001 , respectively. Source data are provided in Source Data file. $P$ values: $3 \mathbf{c}($ Satb2)-<0.0001, (NeuroD1)0.0003, (NeuroD2)-0.003, (Tubb3)-0.004, (Map2)-0.009, (Dcx)-<0.0001, (NeuroG1)-0.003, (Gfap)-0.0005, (Olig1)-0.02, (Olig2)-0.01, (Cd44)-0.04, (Pdgfra)<0.0001, (Cspg4)-0.03; 3d-0.01; 3e-0.002; 3f-0.0001; 3g-0.02.

Ybx1 suppresses H3K27me3 levels in brain NPCs. Given that we detected an interaction between Ybx1 and PRC2, we investigated whether Ybxl affects the genomic distribution of H3K27me3, which is deposited by PRC2, and H3K4me3 by lowinput ChIP-seq ${ }^{43}$ and CUT\&RUN-seq ${ }^{42}$ in Ybx1-KO and sibling control NPCs (Fig. 4a). H3K27me3 and H4me3 levels were similar at Actb locus in control and Ybx1-KO NPCs (Supplementary Fig. S4f). H3K27me3 levels were markedly higher at select forebrain lineage genes including Foxg1, Neurod2, and Satb2 in Ybx1-KO versus control NPCs, whereas H3K4me3 levels were unchanged (Fig. 4c). These were downregulated in $Y b x 1-\mathrm{KO}$ NPCs and bound by Ybx1 in control NPCs. Hox C genes were bound by $\mathrm{Ybxl}$ and displayed higher levels of H3K27me3 in Ybx1-KO vs. control NPCs (Supplementary Fig. S4g), but this did not strongly correlate with gene expression changes. H3K27me3 levels were unchanged and $\mathrm{H} 3 \mathrm{~K} 4 \mathrm{me} 3$ levels increased at genes including Hmga2 and Lin28a in Ybx1-KO vs. control NPCs; these genes were bound by Ybx1 and upregulated in Ybx1-KO NPCs (Supplementary Fig. S4h).

Genome wide, H3K27me3 ChIP-seq signals were higher in Ybx1-KO than those in control NPCs (Fig. 4a, d). The observed increases in H3K27me3 ChIP-seq signals in Ybx1-KO NPCs were validated by H3K27me3 CUT\&RUN-seq (Supplementary Fig. S5a). However, the distribution of H3K27me3-occupied regions was largely the same in control and Ybx1-KO NPCs (Supplementary Fig. S5b, c), suggesting that few H3K27me3occupied regions occurred de novo in Ybx1-KO NPCs. There was a strong correlation between downregulated gene expression and increased levels of $\mathrm{H} 3 \mathrm{~K} 27 \mathrm{me} 3$ (nearly passing a more stringent FDR criterion; Fig. 4d, e, Supplementary Fig. S5d), suggesting that Ybx1 reduces $\mathrm{H} 3 \mathrm{~K} 27 \mathrm{me} 3$ levels to promote gene expression. We concluded that Ybx1 attenuates the deposition of H3K27me3 to promote the expression of genes involved in forebrain specification and neuronal differentiation. 
a — cat. 1 - cat. 2 - cat. $3-$ cat. $4-$ cat. 5
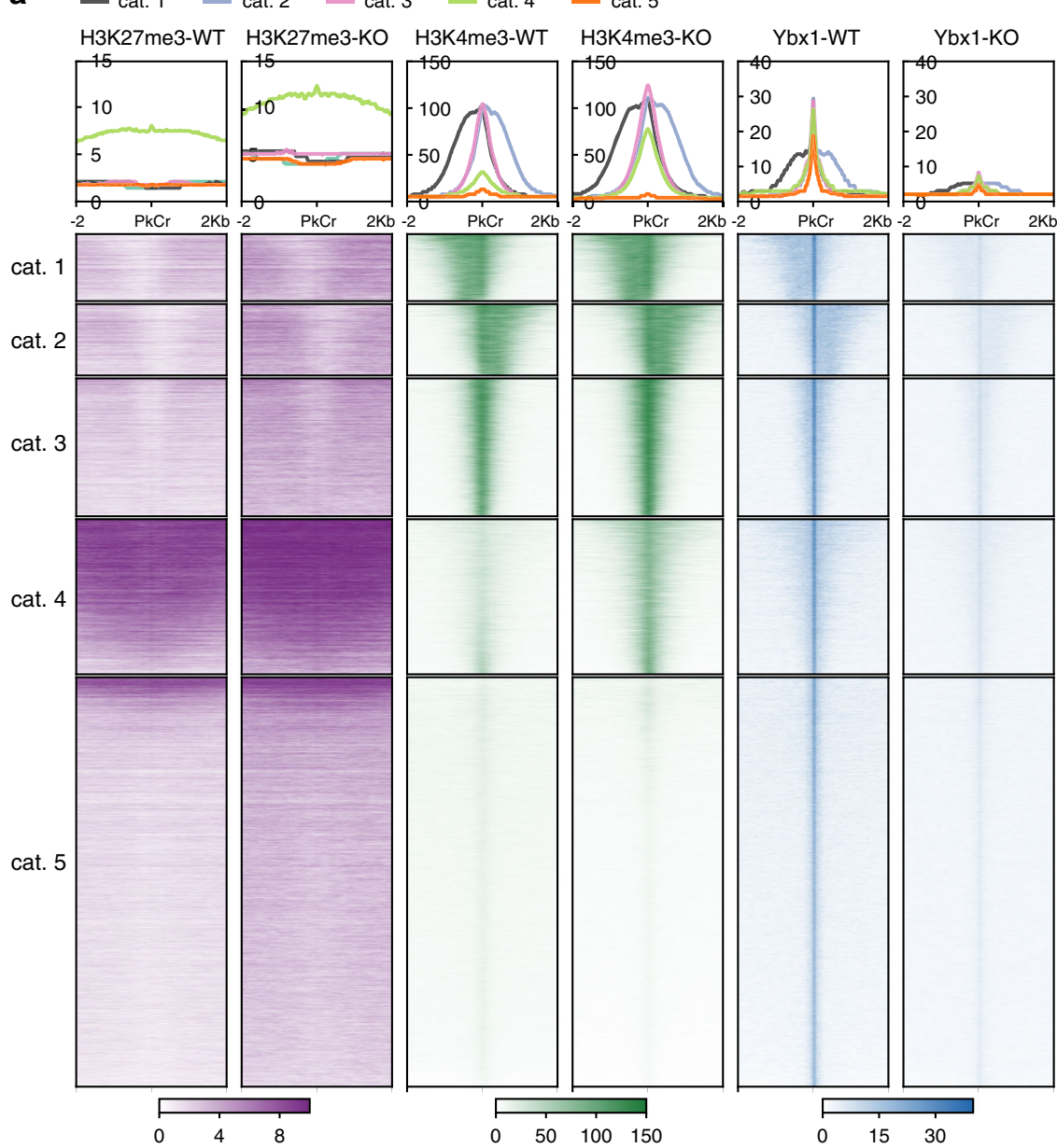

C

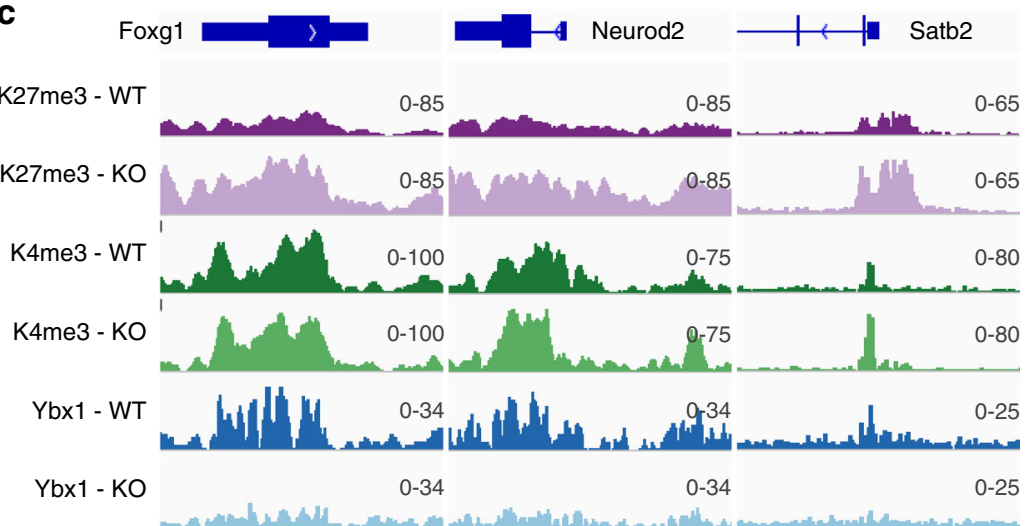

b
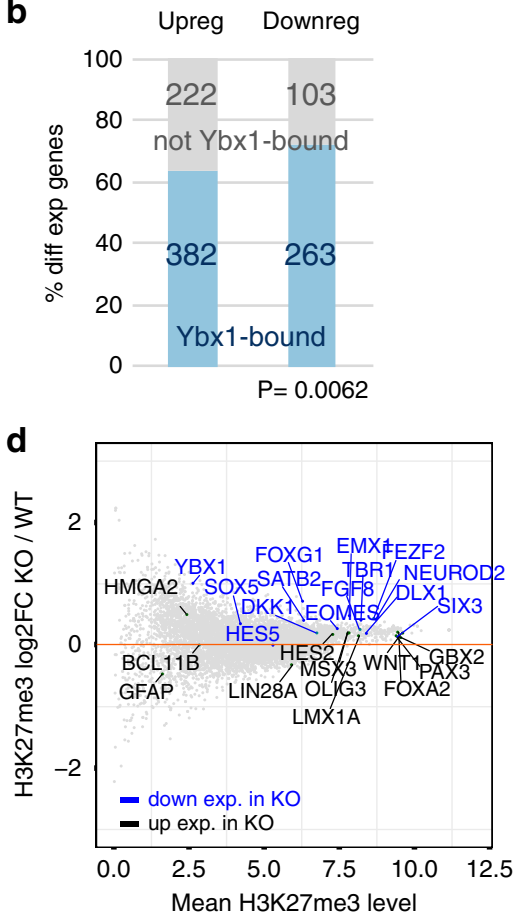

e
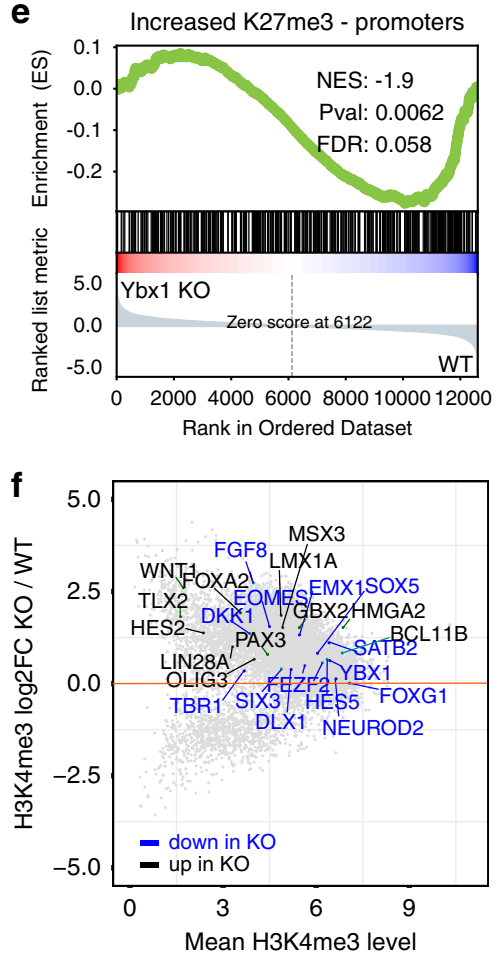

Fig. 4 Ybx1 suppresses H3K27me3 levels. a Heat maps indicate the binding intensity of H3K27me3, H3K4me3, and Ybx1, with low (white) or high (dark color) intensity, within $2 \mathrm{~kb}$ of all Ybx1 CUT\&RUN-seq peaks. Average binding profiles from five different categories (cat.) are presented. In all five categories, H3K27me3 levels increased in Ybx1-KO NPCs. Cat. 1, H3K4me3 peaks to the left of Ybx1 peaks; 2, H3K4me3 peaks to the right of Ybx1 peaks; 3 , H3K4me3 peaks at Ybx1-bound peak center; 4, high H3K4me3 increase in Ybx1-KO; 5, no H3K4me3. b Proportion of differentially expressed genes in Ybx1KO vs. WT NPCs bound by Ybx1 protein in control NPCs. $P$ values were calculated by two-tailed Fisher's exact test. c H3K27me3 and H3K4me3 ChIP-seq and Ybx1 CUT\&RUN-seq tracks at Foxg1, Neurod2, and Satb2 gene loci in Ybx1-KO and sibling control NPCs. d MA plot of LOG2(H3K27me3 fold-changes of Ybx1-KO/WT NPCs) versus mean H3K27me3 levels at individual genes. Blue indicates downregulated genes and black indicates upregulated genes in Ybx1-KO NPCs. e GSEA of genes containing significantly increased H3K27me3 levels at promoters with differentially expressed genes in Ybx1-KO versus control NPCs. $P$ values were calculated by one-tailed Kolmogorov-Smirnov statistic test. $\mathbf{f}$ MA plot is similar to d, but shows H3K4me3 levels. 
We separated Ybx1-bound regions into five categories by intensity and location of $\mathrm{H} 3 \mathrm{~K} 27 \mathrm{me} 3$ and $\mathrm{H} 3 \mathrm{~K} 4 \mathrm{me} 3$ distribution (relative to Ybxl peaks) in Ybx1-KO NPCs, as shown by different average profiles at the top of the heat map in Fig. 4a. (To facilitate explanation, profiles were separated by categories in Supplementary Fig. S5e). For example, H3K27me3 and H3K4me3 levels increased at Ybx1-bound regions in category 4 in $Y b \times 1-\mathrm{KO}$ NPCs. H3K4me3 ChIP-seq signals genome-wide were higher in Ybx1-KO than in control NPCs (Fig. 4f), but the numbers of $\mathrm{H} 3 \mathrm{~K} 4 \mathrm{me} 3$-occupied regions at promoters $3^{\prime}$ of TSS, exons, introns, termination sites, distal regions (within $50 \mathrm{~kb}$ of a coding gene), and intergenic regions all decreased in Ybx1-KO NPCs (Supplementary Fig. S5f, g). Although genes (regardless of Ybx1 binding status) with higher $\mathrm{H} 3 \mathrm{~K} 4 \mathrm{me} 3$ levels were enriched in upregulated genes in Ybx1-KO NPCs (Supplementary Fig. S5h), we did not observe Ybx1 protein binding with H3K4 methyltransferases, which are represented by the core subunit Rbbp5 (Supplementary Fig. S5i). These data suggest that Ybx1 does not directly influence $\mathrm{H} 3 \mathrm{~K} 4 \mathrm{me} 3$.

We next analyzed $\mathrm{H} 3 \mathrm{~K} 27 \mathrm{me} 3$ and $\mathrm{H} 3 \mathrm{~K} 4 \mathrm{me} 3$ distributions at TSSs of Ybxl-bound genes that were unchanged, downregulated, or upregulated in Ybx1-KO NPCs (Supplementary Fig. S5j). As an additional comparison, we generated the same profiles at genes that were not bound by Ybx1; they had little change in $Y b x 1-\mathrm{KO}$ NPCs (Supplementary Fig. S5k). H3K27me3 levels increased by approximately 2 fold at all Ybx1-bound TSSs. WB analysis of neural tube cells showed that H3K27me3 levels were higher in Ybx1-KO vs. control (Supplementary Fig. 5l). We concluded that Ybx1 suppresses H3K27me3 distribution genome-wide.

Ybx1 preferentially binds PRC2-bound genomic features. To investigate possible mechanistic activities of Ybx1, we examined genomic features PRC2 preferentially binds. Nearly all Ybx1bound regions (99.6\%) and TSSs (99.8\%) in categories 1-4 overlapped $\mathrm{CpG}$ islands (Fig. 5a, Supplementary Fig. S6a). However, $44.9 \%$ of Ybx1-bound regions and 30.5\% of Ybx1bound TSSs in category 5 overlapped CpG islands (Fig. 5a, Supplementary Fig. S6a). This finding suggests that Ybx1 binds CpG islands.

We investigated whether Ybx1 binds to bivalent regions, which are co-occupied by $\mathrm{H} 3 \mathrm{~K} 27 \mathrm{me} 3$ and $\mathrm{H} 3 \mathrm{~K} 4 \mathrm{me} 3$. Using the 1-nt overlap criterion, we determined that Ybx1 bound to 4344/7953 (54.6\%) of bivalent regions in NPCs (Fig. 5b). This strong enrichment prompted us to determine whether $Y b x 1 \mathrm{KO}$ led to differential expression of bivalent genes. Compared with sibling control NPCs, 179 bivalent genes were downregulated and 212 bivalent genes were upregulated in Ybx1-KO NPCs (Supplementary Fig. S6b), suggesting Ybxl affects the expression of bivalent genes. As transcription at bivalent genes is generally low and not expected to be downregulated, we examined RNA-seq profiles of some of the 179 bivalent genes. At many of these genes, RNA-seq reads were restricted to the TSSs in control NPCs and reduced further in Ybx1-KO NPCs.

We next examined a potential association of Ybxl with enhancer elements, including active enhancers co-occupied by $\mathrm{H} 3 \mathrm{~K} 4 \mathrm{me} 1$ and H3K27ac and poised enhancers co-occupied by H3K4me1 and H3K27me3. We found that 18.4\% (4235/23070) and $14.5 \%(3352 / 23070)$ of Ybxl-bound regions overlapped active and poised enhancers, respectively. About 12.1\% (4095/ 33934 ) of active enhancers were bound by Ybx1. We found that 29.1\% (3782/9229) of poised enhancers were bound by Ybx1; this higher enrichment suggests a functional association. As our data suggest that Ybx1 suppresses $\mathrm{H} 3 \mathrm{~K} 27 \mathrm{me} 3$ to promote $\mathrm{H} 3 \mathrm{~K} 4 \mathrm{me} 3$ and gene expression, we focused on the 271 Ybxl-bound active enhancers (little to no H3K27me3) in control NPCs that became downregulated in Ybx1-KO NPCs (Supplementary Fig. S6c). Our data suggest that $\mathrm{Ybx} 1$ binds enhancers to promote the expression of genes for nervous system development, synapses, axonogenesis, axon guidance, and dendrite morphogenesis.

Ybx1 promotes PRC2 binding to chromatin but inhibits H3K27me3. We identified a consensus DNA motif for Ybx1 with $p<1 \mathrm{e}-6$ (Supplementary Fig. S6d) in promoters of genes that were upregulated in Ybx1-KO NPCs, consistent with our observation that known Ybxl target genes were upregulated in $Y b \times 1$ KO NPCs (Fig. 2e). On the other hand, of the 382 Ybx1-bound genes that were upregulated in Ybx1-KO NPCs, 132 are known to be bound by PRC2 $(p=2.3 \mathrm{e}-18$ in Suz12 ChIP-seq datasets using ChEA of Enrichr ${ }^{44}$ ), supporting that $\mathrm{Ybxl}$ binds and regulates PRC2-bound genes.

We next examined how Ybxl affects the expression and chromatin binding of PRC2. The expression of PRC2 subunits did not differ between control and Ybx1-KO NPCs (Supplementary Fig. S6e, f). We performed CUT\&RUN-seq of the enzymatic subunits of PRC2, Ezh2/1, in sibling control and Ybx1-KO NPCs (by Sox2-GFP FACS). Ezh2/1 distribution at the Hox A cluster paralleled $\mathrm{H} 3 \mathrm{~K} 27 \mathrm{me} 3$ distribution and remained unchanged in Ybx1-KO NPCs (Supplementary Fig. S6g). Moreover, Ezh2/1 occupancy was significantly lower at the forebrain lineage genes Foxg1, Neurod2, and Satb2 (Fig. 5c) and cell proliferation genes Lin28a and Hmga2 (Supplementary Fig. S6h), whereas H3K27me3 levels were increased. Also, Ezh2/1 localized to an ectopic (only in Ybx1-KO but not in control NPCs) PRC2-bound region that showed increased $\mathrm{H} 3 \mathrm{~K} 27 \mathrm{me} 3$ in Ybx1-KO NPCs (Supplementary Fig. S6i). We concluded that at these genes, PRC2 binding decreased but H3K27me3 levels increased in Ybx1KO NPCs.

Extending our analysis genome-wide with criteria of FDRcorrected $p<0.05$, we identified 43,469 Ezh2/1-bound regions in control NPCs and 10,902 Ezh2/1-bound regions in Ybx1-KO NPCs. Using this criterion, 15,036/43,469 (34.5\%) Ezh2/1-bound regions were found to overlap with Ybx1-bound regions in control NPCs. With a more relaxed criterion of $p<0.05,29,068 /$ 43,469 (66.9\%) Ezh2/1-bound regions were found to overlap Ybxl-bound regions. We generated heat maps to compare Ybx1 and Ezh2/1 distribution at all Ybx1-bound regions (Fig. 5d) or all Ezh2/1-bound regions (Fig. 5e) and showed Ezh2/1 and Ybx1 binding patterns were highly similar and likely overlapped by more than $66.9 \%$ in wild-type NPCs.

Ezh2/1 binding at most regions genome-wide was reduced in Ybx1-KO NPCs compared to control NPCs (Fig. 5e). Ezh2/1 gained binding at ectopic regions (specific to $\mathrm{Yb} x 1-\mathrm{KO}$ in Fig. $5 \mathrm{e}$ ). We could not attribute functional significance to ectopic Ezh2/1bound regions as they were not associated with differentially expressed genes in Ybx1-KO NPCs. H3K27me3 levels increased at all regions with reduced $\mathrm{Ybx} 1$ and Ezh2/1 binding in $Y b x 1-\mathrm{KO}$ NPCs (H3K4me3 remained unchanged; Fig. 5e). At the ectopic Ezh2/1-bound regions in Ybx1-KO, H3K27me3 levels increased and $\mathrm{H} 3 \mathrm{~K} 4 \mathrm{me} 3$ levels remained undetectable (Fig. 5e). Collectively, our data suggest that $\mathrm{Ybx} 1$ binding promotes $\mathrm{PRC} 2$ binding at many sites while restraining its activity including H3K27 methylation. This balancing/fine-tuning of PRC2 binding and histone modifications likely facilitates precise spatiotemporal gene regulation required for neural development.

PRC2 complex physically binds YBX1. We confirmed the JARID2-YBX1 binding with new IP-mass spectrometry datasets using a different JARID2 antibody (R\&D Systems AF6090) in mouse neural stem cells NE-4C (Supplementary Fig. S7a, b). We validated the specificity of the antibody (Supplementary 
a
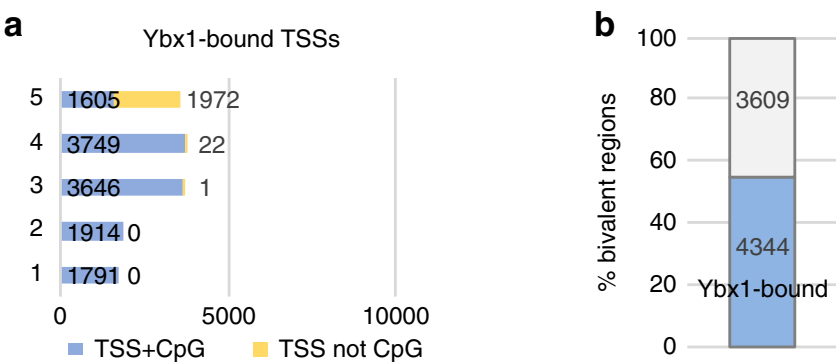

C

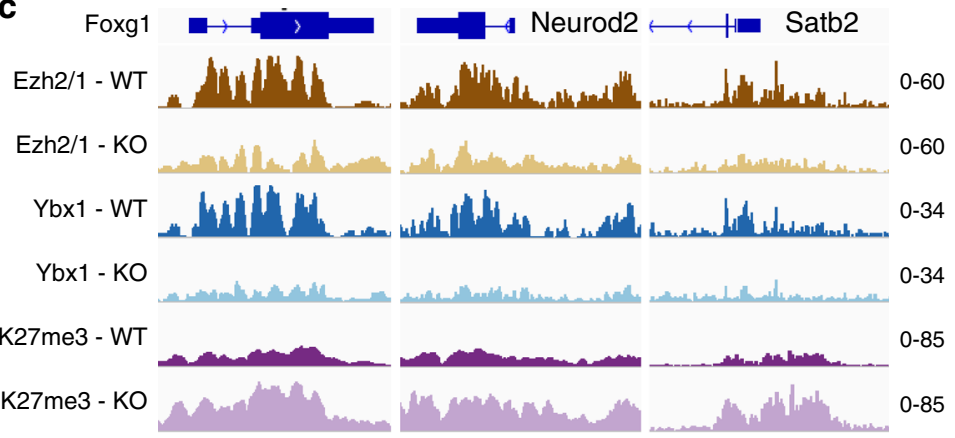

d Ybx1-bound regions
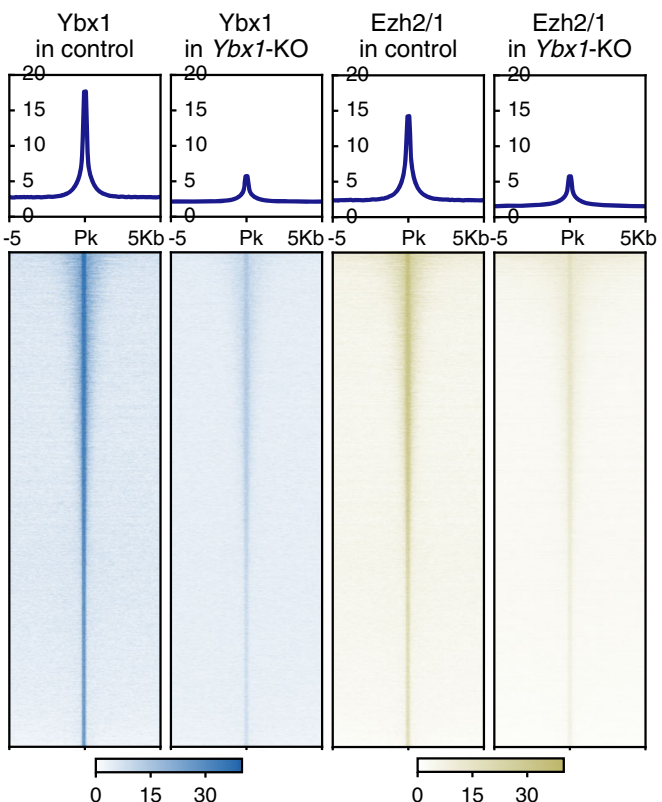

e
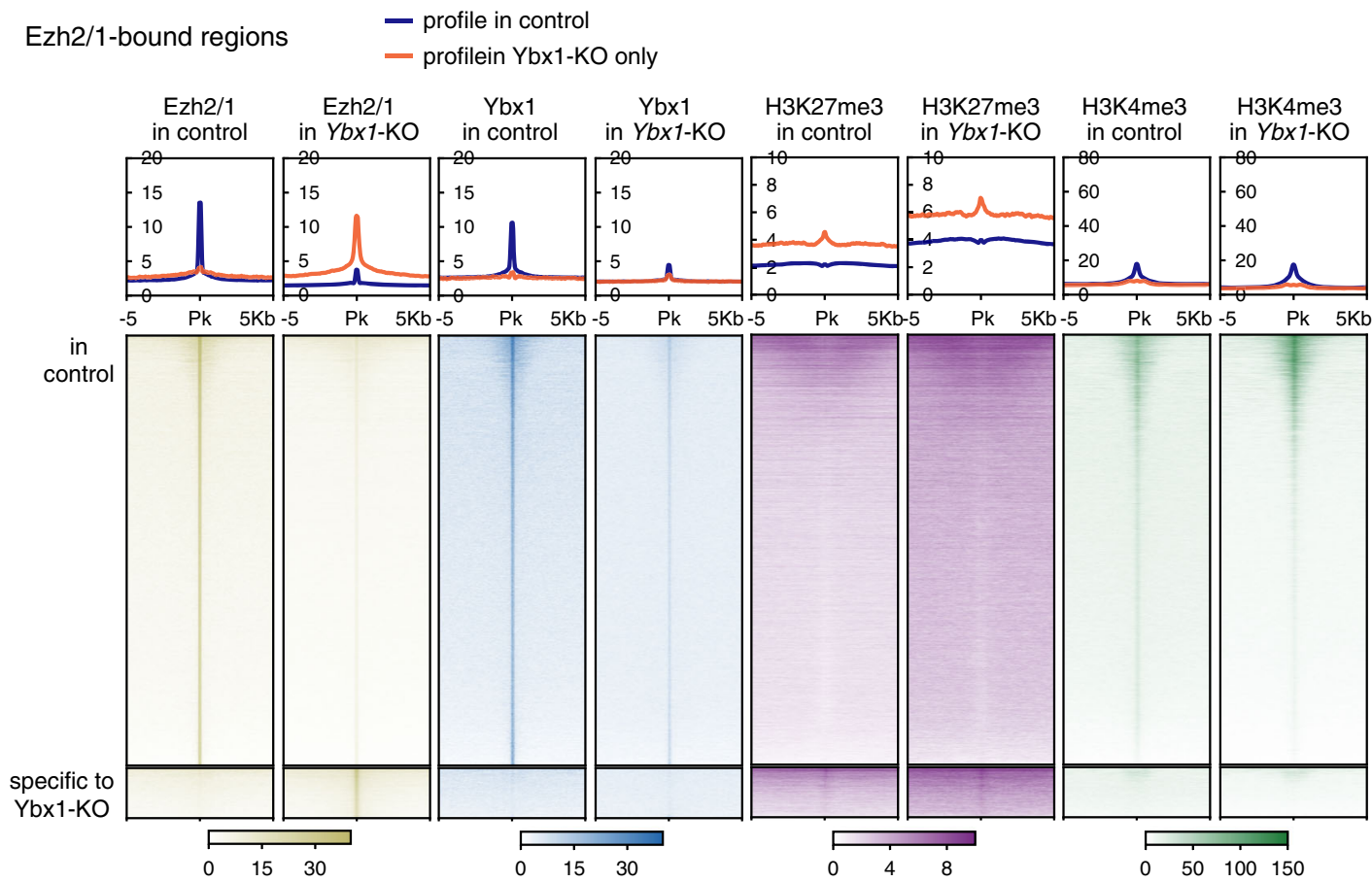

Fig. 5 Ybx1 is required for appropriate levels of PRC2 binding to chromatin. a Proportions of Ybx1-bound TSSs (categories 1-5 based on H3K27me3 and H3K4me3 distribution in Fig. 4) that overlapped CpG islands. b Percent of bivalent (co-occupied by H3K27me3 and H3K4me3) regions that overlap Ybx1bound regions. c Ezh2/1 and Ybx1 CUT\&RUN-seq tracks and H3K27me3 ChIP-seq at Foxg1, Neurod2, and Satb2 gene loci in Ybx1-KO and sibling control NPCs. d Heat maps of Ybx1 and Ezh2/1 distribution within $5 \mathrm{~kb}$ of Ybx1 CUT\&RUN-seq peaks. e Heat maps of Ezh2/1, Ybx1, H3K27me3, and H3K4me3 within $5 \mathrm{~kb}$ of Ezh2/1 CUT\&RUN-seq peaks.

Figs. S2a, S7c, d). Using the hESC nuclear extract, we then performed additional co-IP experiments using benzonase treatment (eliminates nucleic acids for possibly mediating the JARID2-YBX1 interaction), high-stringency washes, and 2 other JARID2 antibodies (Fig. 6a and Supplementary Fig. S7e-g). The depletion of $\mathrm{Ybx}^{27,29}$ and PRC2 subunits ${ }^{11,15-17}$ results in neurodevelopmental defects, so we examined their interactions in NPCs. We showed that that JARID2, SUZ12, and YBX1 interact in hNPCs (Fig. 6c) and further showed that Jarid2 and Ybx1 interact with each other and PRC2 in mouse neural tubes at E13.5 (Fig. 6d). The enrichment of JARID2 in YBX1 co-IP and YBX1 in SUZ12 coIP were low (Fig. 6c, d), suggesting substoichiometric interaction. Subcellular fractionation showed that Ybxl protein localizes to the cytoplasm, nucleoplasm, and on chromatin (Supplementary Fig. S7h). Pull-down showed that JARID2 protein binds to YBX1 protein (Fig. 6e) and that amino acids 1-104 of YBX1 were required for binding to JARID2 (Fig. 6f). 
a + high-stringency wash
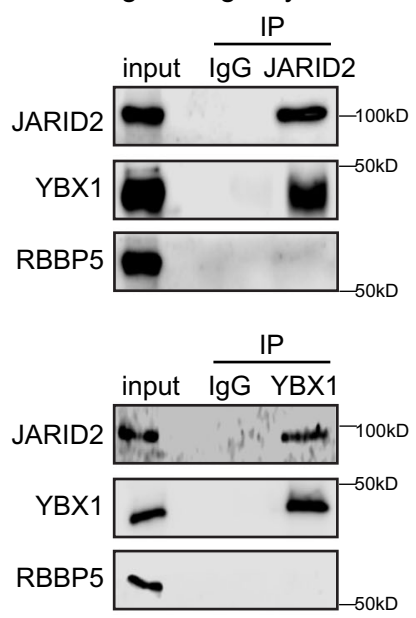

b IP-WB in hNPC

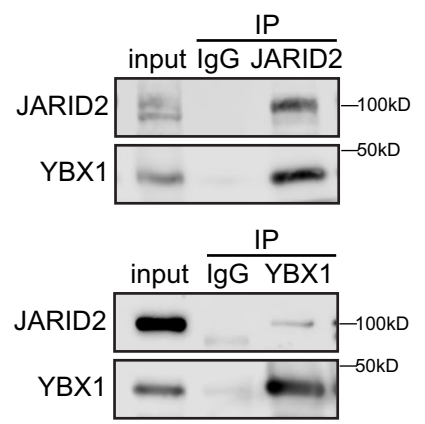

C IP-WB in hNPC

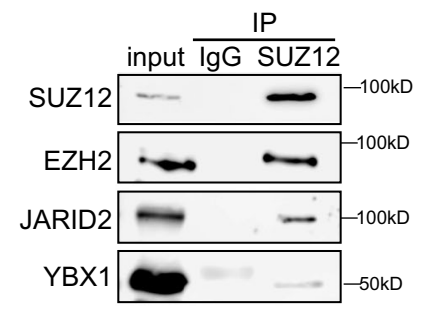

d IP-WB in mouse neural tubes

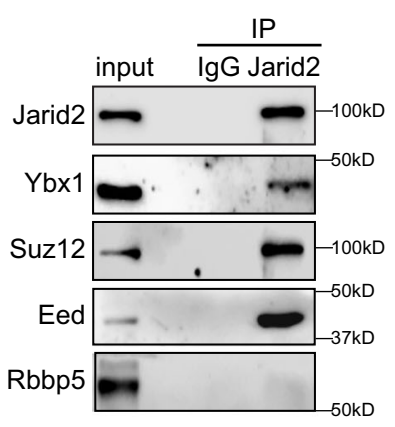

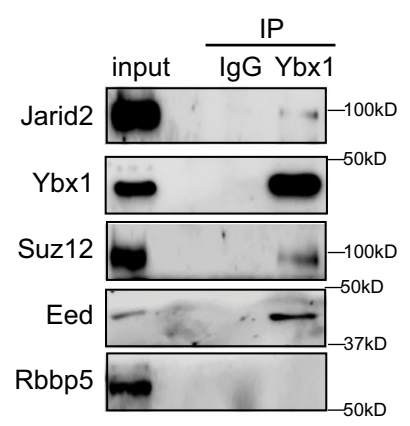

$\mathbf{f}$

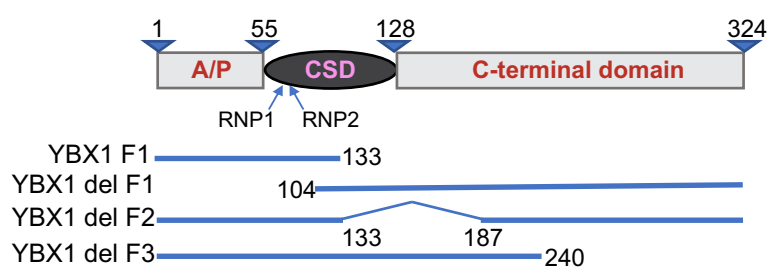

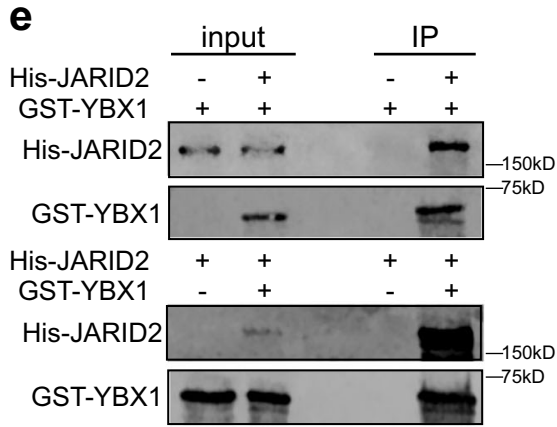

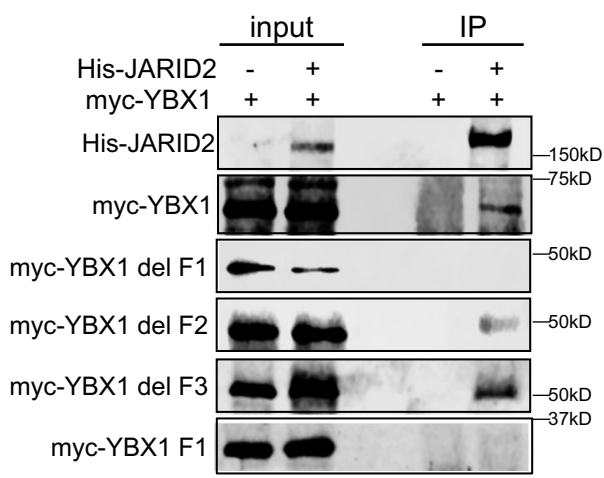

Fig. 6 PRC2 complex binds to Ybx1 in human stem cells and mouse neural tubes. WB analysis of IgG, JARID2, and YBX1 co-IP of a the hESC nuclear extract with high-stringency washes and $\mathbf{b}$ the hNPC nuclear extract. $\mathbf{c}$ WB analysis of IgG and SUZ12 co-IP of the hNPC nuclear extract. $\mathbf{d}$ WB analysis of IgG, Jarid2, and Ybx1 co-IP of the mouse neural tube nuclear extract. WB analysis of His-JARID2 and e GST-YBX1 or $\mathbf{f}$ myc-tagged YBX1 protein fragments. Source data are provided in Source Data file.

Ybx1-mediated regulation of NPCs involves inhibition of H3K27me3. If Ybx1 inhibits H3K27me3 to affect gene expression and, and in turn, NPC self-renewal and differentiation, we predict that $\mathrm{H} 3 \mathrm{~K} 27 \mathrm{me3}$ reduction in Ybx1-KO cells should rescue their defects. To test this, we treated Ybxl-KO NPCs with a specific chemical inhibitor of PRC2, GSK12645, and examined gene expression, proliferation, neurosphere formation, and differentiation (Fig. 7a). Treatment with 100 or $500 \mathrm{nM}$ of GSK126 reduced the global levels of H3K27me3 in Ybx1-KO and sibling control NPCs, as expected (Fig. 7b). Compared with dimethyl sulfoxide (DMSO) treatment, GSK126 treatment of Ybx1-KO NPCs significantly increased the expression of assayed forebrain lineage genes (Fig. 7c and Supplementary Fig. S8a) and decreased the expression of most of the assayed midbrain and hindbrain lineage genes (Supplementary Fig. S8b). GSK126 treatment of control NPCs led to decreased expression of some forebrain lineage genes and increased expression of some midbrain and hindbrain lineage genes (Supplementary Fig. S8c, d). These data suggest that PRC2 inhibition in Ybx1-KO NPCs leads to the reactivation of forebrain lineage genes and suppression of midbrain and hindbrain lineage genes.

We also examined how GSK126 treatment of Ybx1-KO NPCs affects the expression of Cdkn1a and Reln, which are known targets 
a

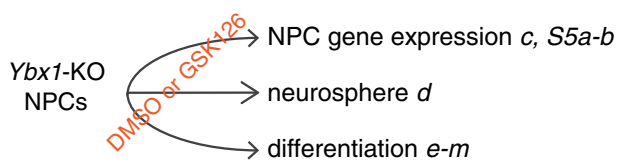

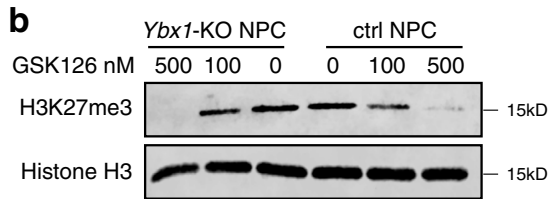

C

GSK + NPC - forebrain genes

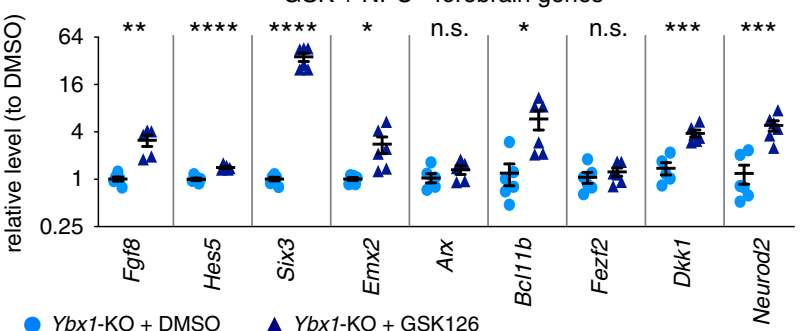

d

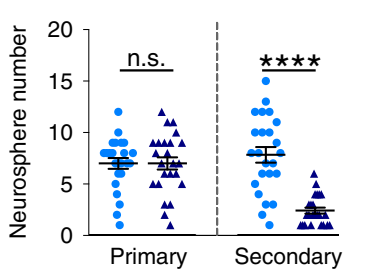

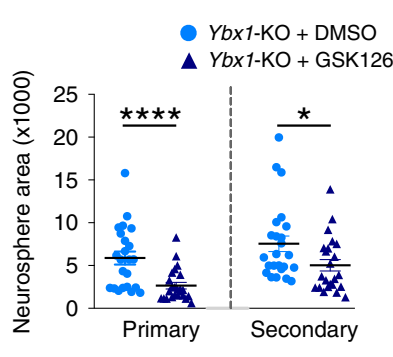

e

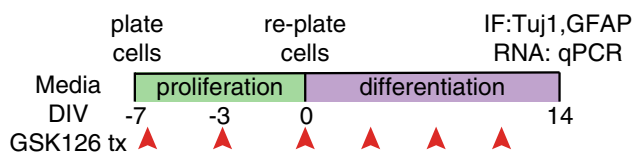

h

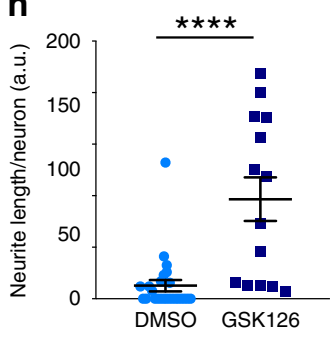

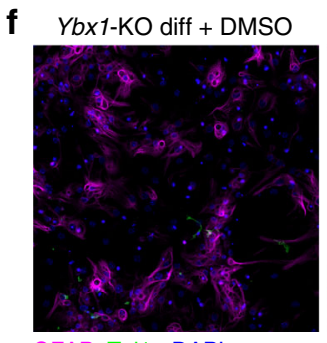

GFAP Tuj1 DAPI

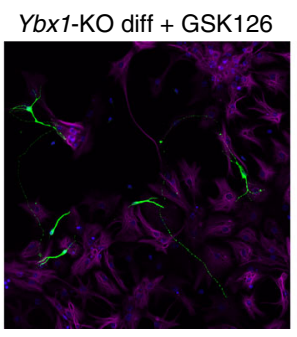

j

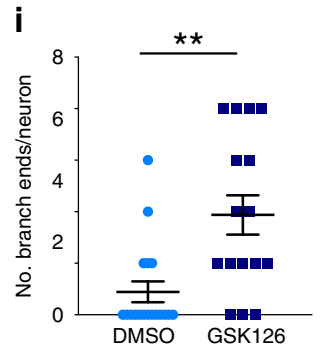

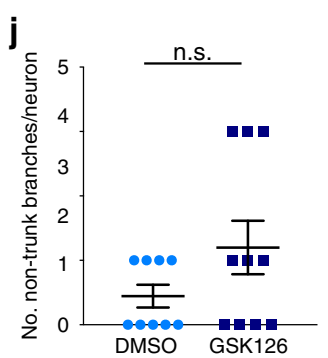
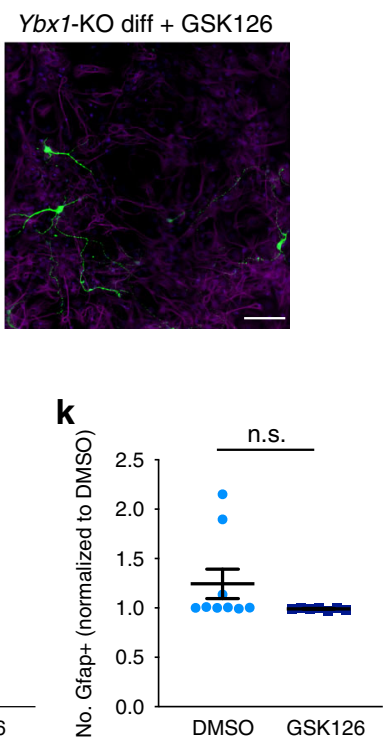

I

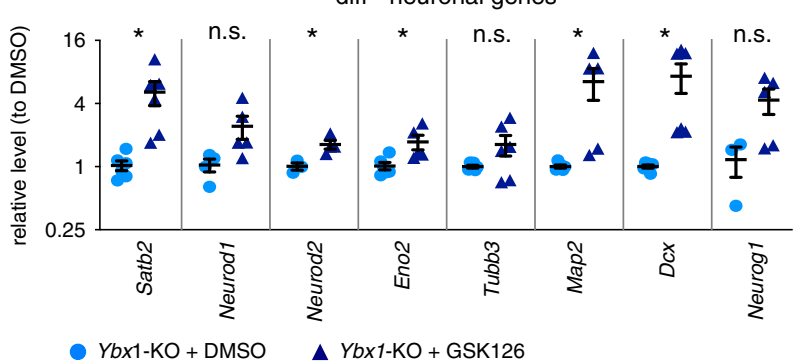

m diff - glial genes

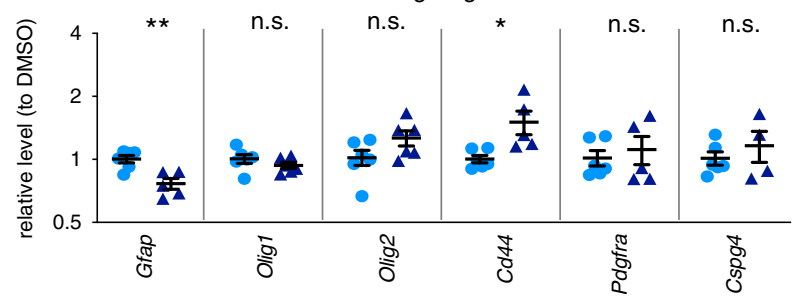

Fig. 7 PRC2 inhibition partially restores the appropriate gene expression, self-renewal, and neuron differentiation in Ybx1-KO NPCs. a Diagram

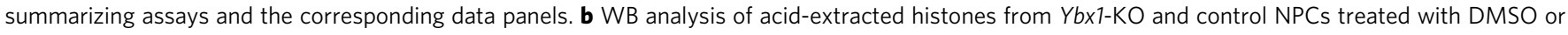

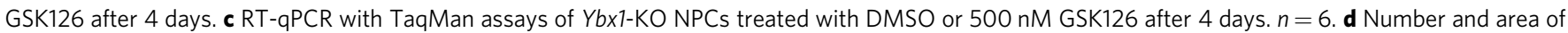
primary and secondary neurospheres formed by Ybx1-KO NPCs pretreated with DMSO or 500 nM GSK126 for 2 days. $n=24$ images/genotype. e Diagram detailing in vitro differentiation of Ybx1-KO NPCs treated with DMSO or 500 nM GSK126. Red arrowheads indicate the days at which DMSO or 500 nM GSK126 was replenished. f IF analysis of Ybx1-KO cells treated with DMSO or 500 nM GSK126 at day 14 of differentiation. Quantification of $\mathbf{g}$ relative number of Tuj1-positive neurons, $\mathbf{h}$ neurite length, $\mathbf{i}$ number of branch points in axons, $\mathbf{j}$ number of non-trunk branches (not connected to an axon or dendrite), and k Gfap-positive (glial marker) cells in Ybx1-KO differentiating cells treated with DMSO or 500 nM GSK126 ( $n=9$ DMSO and 7 GSK126 images with cell numbers indicated). RT-qPCR with TaqMan assays of I neuronal genes and $\mathbf{m}$ glial genes in Ybx1-KO differentiating cells treated with DMSO or $500 \mathrm{nM}$ GSK126. $n=6$, except $n=3 \mathrm{ctrl}$ Neurod1 and Neurog1. Data are presented as mean \pm SEM for $\mathbf{c}$, d, and $\mathbf{g}$-m. $P$ values by two-tailed

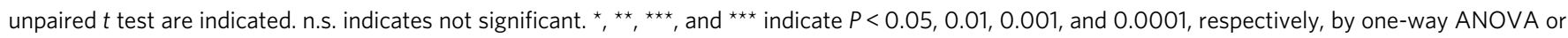
unpaired $t$ test with Holm-Sidak correction. Source data are provided in Source Data file. P values: 7c(Fgf8)-0.002, (Hes5)-<0.0001, (Six3)-<0.0001, (Emx2)-0.02, (Bcl11b)-0.02, (Dkk1)-0.0002 (NeuroD2)-0.0009; 7d(secondary \#)-<0.0001, 7d(primary area)-<0.0001, 7d(secondary area)-0.03; 7g0.02; 7h-<0.0001; 7i-0.001; 7I(Satb2)-0.01, (NeuroD2)-0.03, (Eno2)-0.02, (Map2)-0.02, (Dcx)-0.02; 7m(Gfap)-0.004, (Cd44)-0.02. 
of PRC2 in neural development ${ }^{46-48}$. We found that $C d k n 1 a$ and Reln expression was significantly downregulated in $Y b \times 1-\mathrm{KO}$ compared with sibling control NPCs (Supplementary Fig. S8e), consistent with the loss of Ybx1-mediated inhibition of PRC2. GSK126 treatment led to the upregulation of both Cdkn1a and Reln in wild-type and Ybx1-KO NPCs, as expected due to inhibition of PRC2-mediated gene suppression (Supplementary Fig. S8f).

We examined whether GSK126 affects self-renewal and differentiation of Ybx1-KO NPCs. Compared with DMSO treatment, GSK126 significantly reduced the number and growth of $Y b \times 1-\mathrm{KO}$ neurospheres through serial passages (Fig. 7d). These findings suggest that PRC2 inhibition reduces the self-renewal of $\mathrm{Yb} \times 1-\mathrm{KO}$ NPCs. For the in vitro differentiation assay, we treated $Y b \times 1-\mathrm{KO}$ NPCs with GSK126 before and during differentiation (Fig. 7e). After 14 days of culture in differentiation media, GSK126-treated Ybx1KO cells formed more Tuj1-positive neurons that had longer neurites and more neurite branching (Fig. 7f-j). GSK126 treatment did not significantly affect the number of Gfap-positive glial cells (Fig. 7k), suggesting that glial differentiation was unaffected. GSK126 treatment led to increased expression of some neuronal genes and less effect on glial genes in differentiating Ybxl-KO cells (Fig. 7l, m). GSK126 treatment of differentiating control cells largely had modest to little effect on the expression of most neuronal and glial genes (Supplementary Fig. S8g). Cdkn1a expression was little affected, and Reln was downregulated (Supplementary Fig. S8h). In a separate differentiation assay, YBX1 re-expression by lentiviral transduction was not sufficient to alter the epigenetic programs and restore neuronal differentiation in Ybx1-KO (Supplementary S9a). Our data suggest that PRC2 inhibition in Ybx1-KO NPCs partially reversed cellular defects and restored neuronal differentiation.

We examined whether depletion of Eed, a PRC2 subunit, affects gene expression or differentiation of Ybx1-KO NPCs (purified by Neurofluor $^{\text {TM }}$ CDr3 FACS). We used Nestin promoter-driven Cre recombinase ${ }^{49}$ to excise floxed $E e d^{50}$ in vivo (Fig. 8a). WB analysis confirmed conditional depletion of Eed and reduction of H3K27me3 levels in Ybx1-KO, Eed-conditional KO NPCs (referred to as Ybx1$\mathrm{KO}$; Eed-cKO) (Fig. 8a). Compared with Ybx1-KO NPCs, Ybx1-KO; Eed-cKO NPCs had significantly higher expression of forebrain lineage genes and lower expression of most midbrain and hindbrain lineage genes (Fig. 8b and Supplementary Fig. S9b, c). These results suggest that, similar to GSK126 treatment, Eed depletion in Ybx1KO NPCs restores brain regionalization.

During in vitro differentiation, Ybx1-KO;Eed-cKO cells yielded significantly more neurons with longer neurites than those in $Y b \times 1$ KO cells (Fig. $8 c-e$ ). Eed depletion did not significantly affect neurite branching or the number of Gfap-positive glial cells (Fig. $8 \mathrm{f}-\mathrm{h}$ ), suggesting that neuron maturation and glial differentiation was less affected. Neither Cdkn1a or Reln expression was markedly affected by Eed depletion in Ybxl-KO differentiation (Supplementary Fig. S9d). Compared with Ybx1-KO, Ybx1-KO;Eed-cKO cells had significantly higher expression of some neuronal genes and decreased expression of some glial genes (Fig. 8i and Supplementary Fig. S9e). Altogether, our results suggest that in NPCs, Ybx1 finetunes PRC2 activities to mediate brain regionalization, modulate self-renewal, and promote neuronal differentiation.

\section{Discussion}

The multifunctional role of Ybx1 in different cellular processes to promote cancer progression is well established ${ }^{22,23}$. However, Ybx1 has been mostly studied in cancer cell lines, and the physiological role of Ybx1 in brain development is relatively less understood. Our study advances the understanding of PRC2 regulation in brain development by uncovering that Ybx1 binds to PRC2 to promote PRC2 binding and proper genomic distribution, but inhibits H3K27me3 levels in NPCs. Via PRC2, Ybxl likely influences gene expression for NPC self-renewal modulation and neuronal differentiation (Fig. 8j). Ybxl depletion in the brain leads to reduced forebrain specification concomitant with expanded midbrain and hindbrain regions; these phenotypes are the opposite of those in brains with Ezh2 depletion ${ }^{51}$. We propose the model that Ybx1 regulates $\mathrm{PRC} 2$ to mediate brain regionalization and the decision between self-renewal and neuronal differentiation. This model is strongly supported by the finding that chemical and genetic inhibition of PRC2 partially rescues the defective phenotypes of Ybx1KO NPCs. Taken together, these findings point to a physical and functional interaction between $\mathrm{Ybx} 1$ and $\mathrm{PRC} 2$, such that PRC2 is locked and loaded at co-bound genes in NPCs. An aberrant decrease in PRC2 activity leads to forebrain expansion, whereas an aberrant increase in PRC2 activity in the absence of Ybx1 leads to forebrain reduction. Overall, our data suggest that Ybx1 optimizes PRC2 activity and promotes balanced brain development in vivo.

Because PRC2 and Ybx1 both have cell and development context-dependent influences, their interactions likely differ in forebrain versus mid/hindbrain. The crucial requirement of PRC2 and Ybx1 for spatiotemporal developmental programs, despite their ubiquitous expression, suggests that additional factors within this network provide developmental specificity in neural stem and progenitor cells.

We used in vitro assays for their multiple advantages of validating in vivo studies, extending the investigation of $Y b \times 1-\mathrm{KO}$ beyond the stage of embryonic lethality, showing that PRC2-Ybx1 participates in a cell-intrinsic mechanism to regulate NPCs. Although Ybx1 promotes the proliferation of medulloblastoma or glioblastoma cancer cells ${ }^{25,26}$, it suppresses the proliferation and self-renewal of NPCs. This difference could be due to (i) the dosage effect of Ybx1 from complete genetic depletion in our study versus partial depletion by small interfering RNAs in other studies; (ii) varying activities of Ybx1 in different cell types; and (iii) developmental or pathological contexts of NPCs vs. cancer cells. Indeed, we found that Ybxl suppresses the proliferation of NPCs but promotes the proliferation of MEFs. Future studies on cell type-specific regulation of Ybx1 are needed to fully understand its diverse mechanisms. The PRC2 variant complexes also have cell type-specific molecular activities and influences ${ }^{52}$ that require further study, including whether Ybxl regulates PRC2 in other stem and progenitor cells, or in cancer.

The activities of Ybx1 detailed herein uncover a fundamental mechanism that is crucial for appropriate chromatin dynamics and gene activation during development. Furthermore, Ybx1 likely reaches beyond $\mathrm{H} 3 \mathrm{~K} 27$ methylation, as it has been implicated in influencing P300/CBP and H3K27 acetylation ${ }^{53}$. We hypothesize that $\mathrm{Ybx} 1$ reduces $\mathrm{H} 3 \mathrm{~K} 27 \mathrm{me} 3$ in vivo by inhibiting PRC2 methyltransferase activity, perhaps by reducing complex integrity or stability, and/or binding to RNAs. Alternative mechanistic scenarios include Ybx1 influencing gene activators or chromatin remodelers in order to reduce $\mathrm{H} 3 \mathrm{~K} 27 \mathrm{me} 3$ at different loci. Future identification of other $\mathrm{Ybx} 1$ binding factors, as well as extensive biochemical, genetic, and genomic characterization will be required to pinpoint how Ybx1 affects PRC2 and potentially the activities of other chromatin modifiers.

Patterning and regionalization of the embryonic brain occur in a short developmental time window of 3 days ${ }^{54}$. Polycomb group proteins have emerged as key epigenetic factors that integrate cues from signaling pathways and the transcriptional circuitry to affect gene expression programs underlying brain patterning and regionalization ${ }^{2-4}$. Our findings suggest that $\mathrm{Ybx} 1$ reduces the level of H3K27me3 deposited by PRC2 in NPCs. As ChIP-seq is a population-based assay, $\mathrm{H} 3 \mathrm{~K} 27 \mathrm{me} 3$ reduction potentially occurs in a subset of NPCs. Nevertheless, this reduction appears to be genome-wide and coincides with the Ybx1-dependent expression of PRC2 target genes involved in forebrain lineage specification 
a

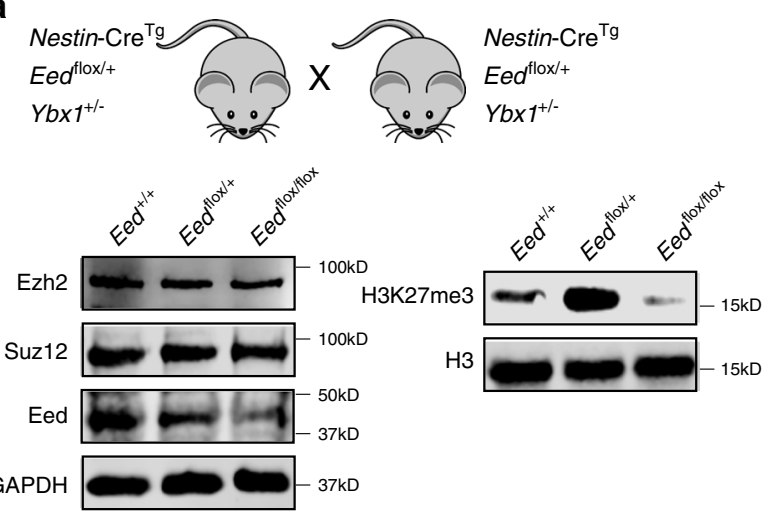

b

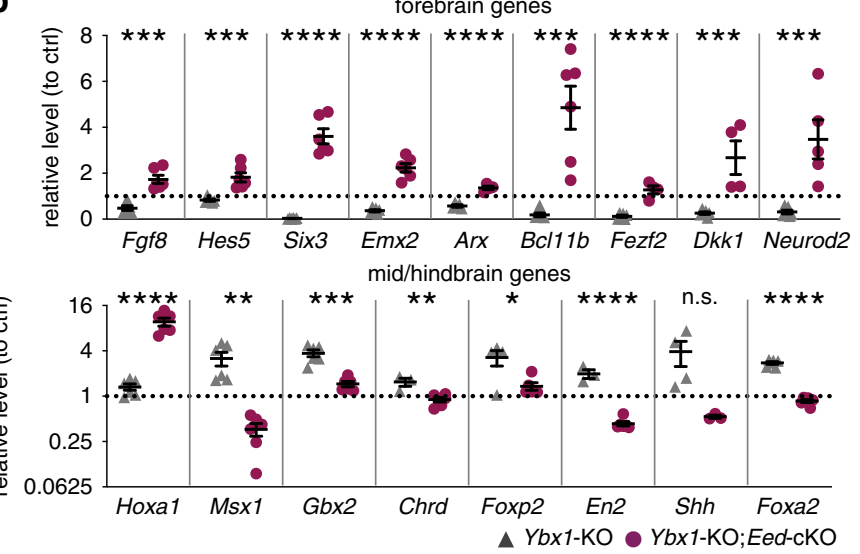

C

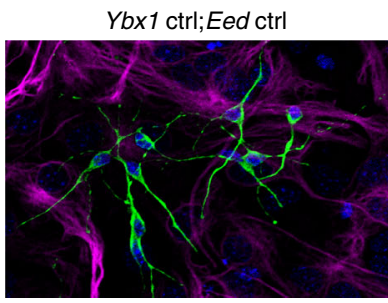

Ybx1 ctrl;Eed cKO
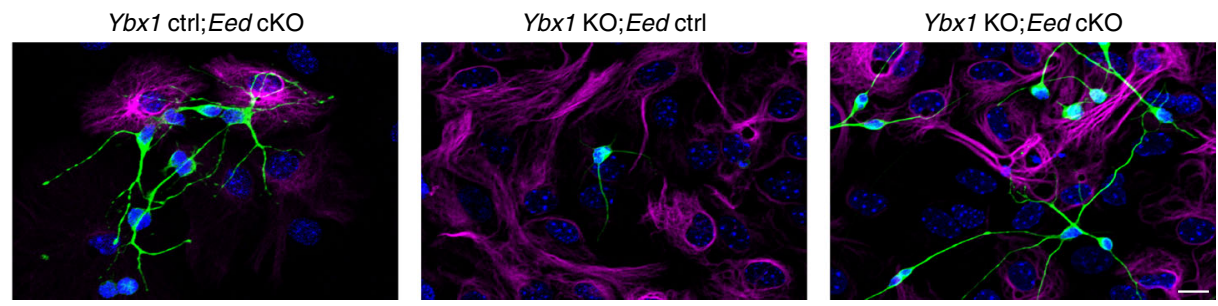

d

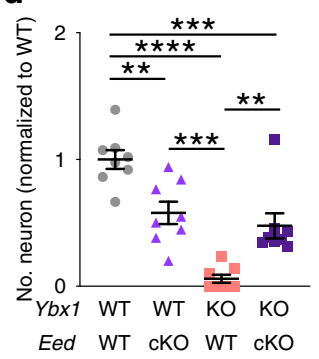

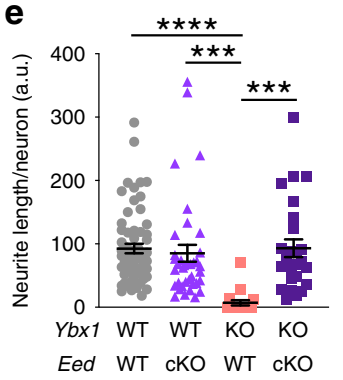

f

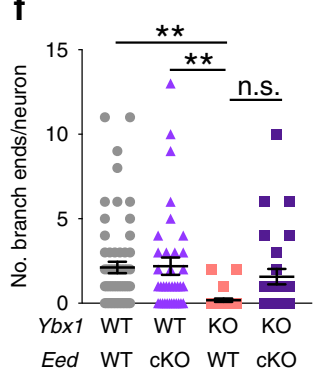

g

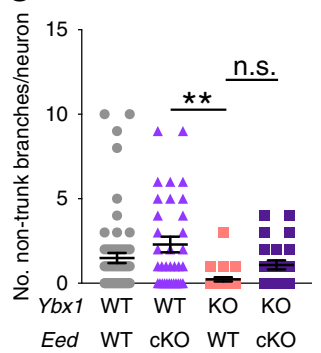

h

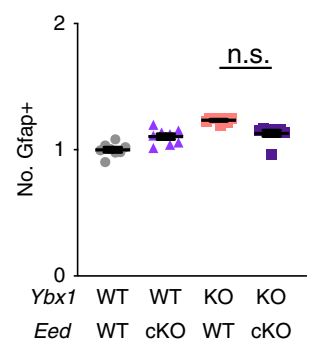

i
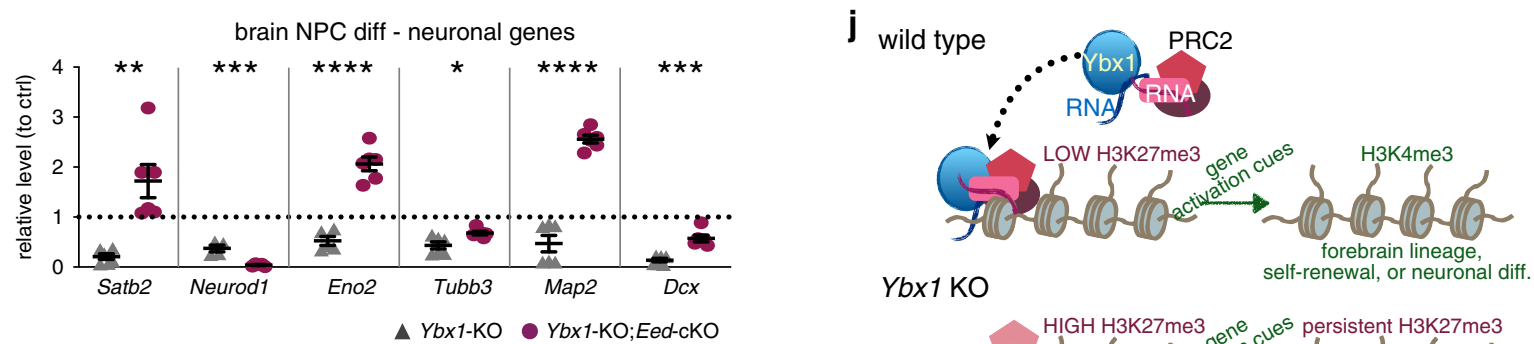

Ybx1 KO

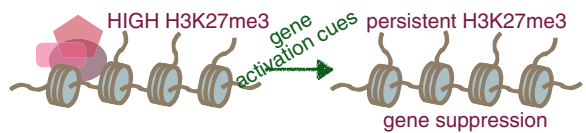

Fig. 8 Depletion of PRC2 subunit Eed partially restores the appropriate gene expression and neuron differentiation in Ybx1-KO NPCs. a Genetic cross scheme to obtain embryos at E13.5 with Ybx1-KO or Ybx1-Eed-cKO by Nestin:Cre. WB analysis of Ybx1-KO NPCs with indicated Eed alleles (all embryos are Nestin:(reTg). b RT-qPCR with TaqMan assays of Ybx1-KO and Ybx1-Eed-cKO. Relative levels were displayed as their standardization to control NPCs. Dotted line indicates the level of gene expression in control cells. $n=6$ except $n \geq 3$ for Chrd, En2, and Shh. c IF analysis of cells with indicated genotypes at day 14 of differentiation. Quantification of $\mathbf{d}$ relative number of Tuj1-positive neurons, e neurite length, $\mathbf{f}$ number of branch points in axons, $\mathbf{g}$ number of non-trunk branches (not connected to an axon or dendrite), and $\mathbf{h}$ Gfap-positive (glial marker) cells in differentiating cells ( $n=8$ images). i RT-qPCR with TaqMan assays of neuronal genes in cells after 14 days of differentiation $(n=6)$. Dotted line indicates the level of gene expression in control cells. $\mathbf{j}$ Model of Ybx1 activities and its influence over PRC2 to ensure spatiotemporal precision in gene activation. Data are presented as mean \pm SEM for $\mathbf{b}$, $\mathbf{d}-\mathbf{i}$. $P$ values by two-tailed unpaired $t$ test are indicated for $\mathbf{b}$ and $\mathbf{i}$ and by ordinary one-way ANOVA followed by Tukey's post hoc test in $\mathbf{d}-\mathbf{h}$. n.s indicates not significant. ${ }^{\star},{ }^{\star \star \star}$, and ${ }^{\star \star \star \star}$ indicate $P<0.05,0.001$, and 0.0001 , respectively. Source data are provided in Source Data file. $P$ values: $8 \mathbf{b}($ Fgf8)-0.0001, (Hes5)-0.0007, (Six3)-<0.0001, (Emx2)-<0.0001, (Arx)-<0.0001, (Bcl11b)-0.0006, (Fezf2)-<0.0001, (Dkk1)-0.003, (NeuroD2)-0.003, (Hoxa1)<0.0001, (Msx1)-0.002, (Gbx2)-0.0002, (Chrd)-0.005, (Foxp2)-0.02, (En2)-<0.0001, (Foxa2)-<0.0001; 8d(Ybx1 WT-Eed cKO)-0.004, (Ybx1 WT-Ybx1 KO)-<0.0001, (Ybx1WT-dKO)-0.0003, (Eed cKO-Ybx1 KO)-0.0003, (Ybx1 KO-dKO)-0.004; 8e(Ybx1 WT-Ybx1 KO)-<0.0001, (Eed cKO-Ybx1 KO)0.0003, (Ybx1 KO-dKO)-0.0002; 8f(Ybx1 WT-Ybx1 KO)-0.0042, (Eed cKO-Ybx1 KO)-0.008; ;8g(Eed cKO-Ybx1 KO)-0.0013; 8i(Satb2)-0.001, (NeuroD1)-0.0009, (Eno2)-<0.0001, (Tubb3)-0.01, (Map2)-<0.0001, (Dcx)-0.0002. 
and neuronal differentiation. Overall, our findings suggest that modest to low levels of $\mathrm{H} 3 \mathrm{~K} 27 \mathrm{me}$, regulated by Ybx1, enable but also restrain the activation of PRC2 target genes during this short developmental time window.

\section{Methods}

Buffers. PBS: $137 \mathrm{mM} \mathrm{NaCl}, 2.7 \mathrm{mM} \mathrm{KCl}, 10 \mathrm{mM} \mathrm{Na} 2 \mathrm{HPO}_{4}, 1.8 \mathrm{mM} \mathrm{KH}_{2} \mathrm{PO}_{4}$ (pH 7.4). PBS-T: PBS with 0.1\% Triton X-100. HEPM: 25 mM HEPES ( $\mathrm{pH} 6.9$ ) $10 \mathrm{mM}$ EGTA, $60 \mathrm{mM}$ PIPES, $2 \mathrm{mM} \mathrm{MgCl}_{2}$. IF blocking solution: $1 / 3$ Blocker Casein (ThermoFisher 37528), 2/3 HEPM with 0.05\% TX-100. Buffer A: $10 \mathrm{mM}$ HEPES (pH 7.9), $10 \mathrm{mM} \mathrm{KCl}, 1.5 \mathrm{mM} \mathrm{MgCl} 2,0.34 \mathrm{M}$ sucrose, $10 \%$ glycerol. Buffer B: $3 \mathrm{mM}$ EDTA, $0.2 \mathrm{mM}$ EGTA. Buffer D: $400 \mathrm{mM} \mathrm{KCl}, 20 \mathrm{mM}$ HEPES, $0.2 \mathrm{mM}$ EDTA, 20\% glycerol. FACS antibody staining solution: PBS containing $0.5 \%$ Tween $20,1 \%$ bovine serum albumin, and $0.5 \mu \mathrm{g} / \mu \mathrm{L}$ RNase. CUT\&RUN Binding buffer: $20 \mathrm{mM}$ HEPES-KOH (pH 7.9), $10 \mathrm{mM} \mathrm{KCl}, 1 \mathrm{mM} \mathrm{CaCl}$, and $1 \mathrm{mM} \mathrm{MnCl}$. CUT\&RUN Wash buffer: $20 \mathrm{mM}$ HEPES pH 7.5, $150 \mathrm{mM} \mathrm{NaCl}, 0.5 \mathrm{mM}$ spermidine, protease inhibitor (PI). CUT\&RUN Digitonin block buffer: Wash buffer with $2 \mathrm{mM}$ EDTA, $0.05 \%$ digitonin. CUT\&RUN Stop Buffer: Into $1 \mathrm{~mL}$ of Digitonin block buffer, add $5 \mu \mathrm{L}$ of $10 \mathrm{mg} / \mathrm{mL}$ RNase A and $133 \mu \mathrm{L}$ of $15 \mathrm{mg} / \mathrm{mL}$ GlycoBlue. High-stringency wash buffer: $15 \mathrm{mM}$ Tris- $\mathrm{HCl}$ (pH 7.5), $5 \mathrm{mM}$ EDTA (pH 8.0), $2.5 \mathrm{mM}$ EGTA, $1 \%$ Triton X-100, $1 \%$ sodium deoxycholate, $0.1 \%$ SDS, $120 \mathrm{mM} \mathrm{NaCl}, 25 \mathrm{mM} \mathrm{KCl}$.

Tissue culture cells. H9/WA09 (WiCell) cells were grown on Matrigel with reduced growth factors (ThermoFisher Scientific 354230) in mTeSR1 medium (STEMCELL Technologies 85850) at $37^{\circ} \mathrm{C}$ and $5 \% \mathrm{CO}_{2}$. NE4-C (ATCC CRL-2925; mouse neural stem cell line) cells were grown on Poly-L-lysine $(10 \mu \mathrm{g} / \mathrm{mL}$; Sigma P9155) in Eagle's Minimum Essential Medium (ATCC 30-2003) supplemented with $2 \mathrm{mM}$ L-Glutamine (Invitrogen 25030-081) and 10\% fetal bovine serum (Fisher Scientific 16000044 ) at $37^{\circ} \mathrm{C}$ and $5 \% \mathrm{CO}_{2}$.

Antibodies. Supplementary Table S1 lists all antibodies and conditions used in this study.

Co-immunoprecipitation. For co-immunoprecipation, subcellular fractionation was performed as above, but Buffer D + PI + DTT was substituted for Buffer B and the nuclear fraction was diluted with an equal volume $\mathrm{H}_{2} \mathrm{O}+\mathrm{PI}+$ DTT.

Approximately $1.5 \mathrm{mg}$ of the nuclear extract was incubated with primary antibody overnight at $4{ }^{\circ} \mathrm{C}$. Extract and antibody were added to protein $\mathrm{A}$ and protein $\mathrm{G}$ Dynabeads $^{\text {tw }}$ (ThermoFisher 10002D and 10004D) for $5 \mathrm{~h}$ at $4{ }^{\circ} \mathrm{C}$, washed with PBS-T, and eluted with $0.1 \mathrm{M}$ glycine ( $\mathrm{pH}$ 2.3). Eluates were neutralized with $1 / 10$ volume of $1.5 \mathrm{M}$ Tris buffer ( $\mathrm{pH}$ 8.8). For benzonase, nuclear extracts were treated with $125 \mathrm{U} / \mathrm{mL}$ benzonase (Sigma) for $30 \mathrm{~min}$ at RT before addition of antibody and subsequent IP. High-stringency wash buffer was used in replacement for PBS$\mathrm{T}$ washes in indicated figures.

Subcellular fractionation. Human and mouse NPCs were incubated in a $2 \times$ volume of Buffer A + PI + DTT for 5 min on ice to obtain the cytoplasmic fraction. After centrifugation at $1750 \mathrm{~g}$ for $2 \mathrm{~min}$ at $4{ }^{\circ} \mathrm{C}$, the nuclei pellet was washed in $1 \times$ volume Buffer A and subsequently incubated for $\sim 45 \mathrm{~min}$ in Buffer B + PI + DTT at $4{ }^{\circ} \mathrm{C}$ with rotation to obtain the nuclear fraction. Histones were acid extracted on pelleted chromatin with $0.1 \mathrm{~N} \mathrm{HCl}$ at $4{ }^{\circ} \mathrm{C} \mathrm{O} / \mathrm{N}$. Acid was neutralized with $1 / 10$ volume of $1.5 \mathrm{M}$ Tris buffer ( $\mathrm{pH} 8.8$ ). For whole-cell extracts, cell pellets were incubated directly in Buffer D + PI + DTT.

Mass spectrometry. For each of Supplementary Fig. S1d or S7b, we analyzed three biological replicate samples of JARID2 and IgG IP from the nuclear extract of H9 or NE-4C, respectively. Samples were run on a short gel. Sample slices were digested overnight at $37^{\circ} \mathrm{C}$, acidified and the peptides were extracted in Acetonitrile. Extracts were dried down in a speed vacuum and reconstituted in 5\% Formic acid. Digested peptides were loaded on a nanoscale capillary reverse phase C18 column $(75 \mathrm{id}, 10 \mathrm{~cm})$ by a HPLC system (Thermo Ultimate 3000or EASY-nLC 1000). Buffer A was $0.2 \%$ Formic acid and Buffer B was $70 \%$ Acetonitrile; $0.2 \%$ Formic acid. The peptides were eluted by increasing organic from $12-70 \%$ over a 90-min liquid chromatography gradient. The eluted peptides were ionized by electrospray ionization, and detected by an inline mass spectrometer (Thermo LTQ Orbitrap Elite). The mass spectrometer was operated in data-dependent mode with a survey scan in Orbitrap (240,000 resolution, $1 \times 10^{6}$ AGC target and $200 \mathrm{~ms}$ maximal ion time) and 20 low resolution MS/MS scans in the ion trap (CID, $2 \mathrm{~m} / \mathrm{z}$ isolation width, Normalized collision energy 35 , AGC target $7 \times 10^{4}$, and $250 \mathrm{~ms}$ maximal ion time) for each cycle. The raw data were searched against the UniProt mouse database concatenated with a reversed decoy database for evaluating false discovery rate. Database searches were performed using the Sequest ${ }^{55}$ v.28 (rev. 12) search engine. Searches were performed using a $25 \mathrm{ppm}$ mass tolerance for precursor and $0.5 \mathrm{Da}$ for product ions, fully tryptic restriction with two maximal missed cleavages, three maximal modification sites, and the assignment of $b$, and $y$ ions. Carbamidomethylation of Cysteine $(+57.02146 \mathrm{Da})$ was used for static modifications and Met oxidation $(+15.99492 \mathrm{Da})$ was considered as a dynamic modification. All matched MS/MS spectra were filtered by mass accuracy and matching scores to reduce protein false discovery rate to $<1 \%$. Finally, all proteins identified in one gel lane were combined together. The total number of spectra, namely spectral counts (SC), matching to individual proteins may reflect their relative abundance in one sample after the protein size is normalized ${ }^{56}$. The abundance values (spectral count $\times 50 \mathrm{kD} /$ protein size $\mathrm{kD}$ ) of proteins were used to compared between the replicate datasets by T-test.

Western blotting. Equal amounts of nuclear extracts were separated by SDSPAGE and transferred onto a nitrocellulose membrane (Bio-Rad). Membranes were blocked with $2 \%$ BSA in HEPM, incubated in primary antibodies (HEPM containing $1 \%$ BSA and $0.1 \%$ Triton X-100) overnight at $4^{\circ} \mathrm{C}$, washed in PBS-T, incubated in IRDye ${ }^{\circ}$-conjugated secondary antibodies (LI-COR) or Clean-blot IP detection reagent (Life Technologies 21230), followed by SuperSignal ${ }^{\mathrm{mt}}$ West Pico PLUS Chemiluminescent Substrate (ThermoFisher 34577), and imaged on an Odyssey ${ }^{\circledR} \mathrm{Fc}$ imaging system (LI-COR). Signals were quantitated with the Image Studio $^{\text {mit }}$ software (version 1.0.14; LI-COR). Student's $t$ test was used for statistical analyses.

Animals. All mice were group-housed and maintained in the Animal Resource Center at St. Jude Children's Research Hospital under protocols approved by the Institutional Animal Care and Use Committee. We complied with all relevant ethical regulations for animal testing and research. Mice of both sexes were used for all experiments, and they were analyzed at multiple ages (E12.5-E14.5), as described in the text and legends for each figure. All samples were analyzed relative to littermates. For Eed-flox experiments, all animals were crossed to Nestin-Cre and genotypes are indicated in figures. Details of mouse strains are as follows:

Nestin-Cre: B6.Cg-Tg(Nes-Cre) $1 \mathrm{Kln} / \mathrm{J}$, described in Tronche et al. ${ }^{49}$.

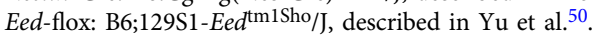

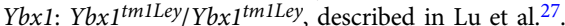

Sox2-eGFP: B6;129S1-Sox $2^{\text {tmlHoch }} / \mathrm{J}$, described in Arnold et al. ${ }^{57}$.

For genotyping, primers are following. Ybx1 WT: F-AGGAACGGATACGGT TTCATCA; R-AGCGGGTCACATTCTTACATAG $\left(54^{\circ} \mathrm{C}, 30 \mathrm{~s}\right)$. Ybx1 null: F- AG GAACGGATACGGTTTCATCA; R- TGAGACGTGCTACTTCCATTT $\left(54^{\circ} \mathrm{C}\right.$, 30 s). Eed flox: F-GGCCCACATAGGCTCATAGA; R- CTACGGGCAGGAGGA AGAG $\left(55^{\circ} \mathrm{C}, 30 \mathrm{~s}\right)$. Nestin-Cre: F-ATGCCCAAGAAGAAGAGGAAGGT; R-GA AATCAGTGCGTTCGAACGCTAGA $\left(56^{\circ} \mathrm{C}, 30 \mathrm{~s}\right)$. Actb: F- ATGTCACGCACG ATTTCCCT, R- TCCCGGGTAACCCTTCTCTT (any AT, 45 s). Sox2-eGFP: F- C GTAAACGGCCACAAGTTCA, R-CTCAGGTAGTGGTTGTCGGG $\left(56^{\circ} \mathrm{C}, 40 \mathrm{~s}\right)$ Reaction products: $354 \mathrm{bp}\left(\mathrm{Ybxl}^{\mathrm{WT}}\right), 477 \mathrm{bp}\left(\mathrm{Ybx}^{\text {null }}\right) ; 200 \mathrm{bp}\left(\mathrm{Eed} \mathrm{d}^{\mathrm{WT}}\right), 400 \mathrm{bp}$

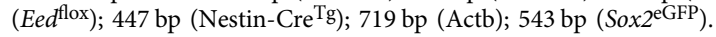

\section{Isolation, culture, and differentiation of Mouse Neural Progenitor Cells}

(mNPCs). Tissue culture plates for adherent culture were prepared by coating them with $10 \mu \mathrm{g} / \mathrm{mL}$ poly-D-lysine (MilliporeSigma P7280) for either $2 \mathrm{~h}$ at room temperature (RT) or at $4{ }^{\circ} \mathrm{C} \mathrm{O} / \mathrm{N}$ (overnight). Plates were rinsed twice with $1 \times \mathrm{PBS}$ and replaced with $10 \mu \mathrm{g} / \mathrm{mL}$ laminin (MilliporeSigma L2020) for $2 \mathrm{~h}$ at RT. As laminin is highly sensitive to drying, it was removed well-by-well and immediately replaced with sufficient culture medium to cover the surface of the well.

Cultures of mouse neural progenitor cells (mNPCs) were derived from E12.5-E13.5 mice as follows. Neural tubes were dissected from embryos in ice-cold Advanced DMEM culture media (ThermoFisher 2491015) and dissociated with 1/3 volume of Type II collagenase $(10 \mathrm{mg} / \mathrm{mL}$, Worthington LS004176) for $10 \mathrm{~min}$ at $37^{\circ} \mathrm{C}$. The tissue was washed once in $1 \times$ PBS and incubated in $500 \mu \mathrm{L} 0.25 \%$ Trypsin-EDTA (ThermoFisher 25200056) for $5 \mathrm{~min}$ at $37^{\circ} \mathrm{C}$. Trypsinization was halted using $500 \mu \mathrm{L}$ DMEM $+10 \%$ fetal bovine serum, and cells were mechanically dissociated by trituration and then pelleted by centrifugation at $1000 \mathrm{~g}$ for $3 \mathrm{~min}$. Cells were resuspended in $1 \mathrm{~mL}$ of PBS and filtered through a $40 \mu \mathrm{m}$ filter (ThermoFisher 22363547) to obtain single cells. NPCs were isolated by fluorescence-activated cell sorting (FACS) using Sox2-eGFP or NeuroFluor CDr3 (STEMCELL Technologies, \#01800), resuspended in complete culture medium, and grown on ultra-low attachment six-well plates. After sphere formation, spheres were dissociated with Accutase (STEMCELL Technologies 07920) for $5 \mathrm{~min}$ in the incubator and single cells plated on coated plates.

Cells were grown in NeuroCult ${ }^{\mathrm{m}}$ Proliferation Media (STEMCELL Technologies 05702) supplemented with $10 \mu \mathrm{g} / \mathrm{mL}$ rhEGF (STEMCELL Technologies 78006) to a final concentration of $20 \mathrm{ng} / \mathrm{mL}$. For routine passaging, cells were grown to $75-80 \%$ confluency and then dissociated using Accutase for $5 \mathrm{~min}$ in the incubator. Cells were then diluted with an equal volume of growth medium and dissociated by trituration. Cells were pelleted as described above and resuspended in fresh culture medium to plate at recommended densities. For differentiation, cells were plated at recommended densities on poly-D-lysine/laminin-coated six-well plates or Matrigel (ThermoFisher 354240)-coated eight-well chamber slides (EMD Millipore PEZGS0816) and left $\mathrm{O} / \mathrm{N}$. Cells were washed once in Neurocult $\mathrm{t}^{\mathrm{m}}$ Proliferation Media without EGF and incubated in Neurocult $\mathrm{t}^{\mathrm{m}}$. Differentiation media (STEMCELL Technologies 05704) for indicated times are detailed in figure legends. 
BrdU administration. Mice were administered 5-bromo- $2^{\prime}$-deoxyuridine (BrdU, MilliporeSigma) reconstituted in sterile PBS by intraperitoneal injection at a dose of $50 \mathrm{mg} / \mathrm{kg}$. For cell culture, cells were treated with $10 \mu \mathrm{M}$ BrdU for indicated times at $37^{\circ} \mathrm{C}$.

Immunofluorescence. Cells and tissues were blocked with IF blocking solution for $2-3 \mathrm{~h}$ at RT and primary antibodies (diluted in blocking buffer) added and incubated $\mathrm{O} / \mathrm{N}$ at $4{ }^{\circ} \mathrm{C}$. After $3 \mathrm{X}$ wash in PBS-T, fluorescent dye-conjugated secondary antibodies (1:500, Alexa Fluor antibodies from ThermoFisher) were added and incubated for $3 \mathrm{~h}$ at RT. Secondary was washed with PBS-T three times and tissues incubated in DAPI (1:1000, ThermoFisher) for $5 \mathrm{~min}$ and coverslips mounted with Prolong Gold Antifade Mountant (ThermoFisher P36930). Cells were washed and coverslips mounted with Prolong Glass Mounting Reagent (Thermo Fisher P36981) which contains DAPI.

For BrdU detection, antigen retrieval was performed using $\mathrm{HCl}$. Slides were thawed and rinsed in PBS for $5 \mathrm{~min}$ at RT. Samples were incubated in fresh $2 \mathrm{~N}$ $\mathrm{HCl}$ for $1 \mathrm{hr}$ or $30 \mathrm{~min}$ at RT. Samples were rinsed through multiple PBS washes to remove all traces of $\mathrm{HCl}$. For combination IF with SOX2 or YBX1, antibodies were tested for affinity post- $\mathrm{HCl}$ in a separate experiment and combined with BrdU for experiments. Antibodies were used as follows: Sox2: diluted 1:200. Ybx1: rabbit/ mouse diluted 1:100. BrdU: diluted 1:100. Foxg1: diluted 1:50. Gbx2: diluted 1:100. GFAP: diluted rabbit-1:200, mouse-1:250. Tuj1: diluted 1:100. Images were acquired with Zeiss LSM780 or Keyence BZ-X and image modifications performed with Fiji or Adobe Photoshop.

CellProfiler analysis. For BrdU quantification, CellProfiler was used to directly quantify the number of positive cells in each image. The DAPI-positive cells were enumerated using IdentifyPrimaryObjects, and BrdU- positive cells were identified in relation to those cells by using IdentifyPrimaryObjects and RelateObjects. For IHC sections, the BrdU cell number was divided by the SOX2-positive cell number to account for differences in total cell numbers and mNPC numbers in each section. For Tuj1 quantification and measurements, CellProfiler was used to quantify neuron number and characteristics of neurons. First, color images were transformed to grayscale and neurites enhanced by through two runs of EnhanceorSuppressFeatures: (1) Enhance, Speckles, Feature Size 12, Fast/Hexagonal and (2) Enhance, Neurites, Tubness, Smoothing Scale 1.8. Next, Morph erode, Once, Disk 3.0 was used to erode excess pixels. IdentifyPrimaryObjects with automatic thresholding was used followed by IdentifySecondaryObjects with Adaptive Otsu thresholding (two classes, weighted variance, no smoothing, regularization factor of 0.05). Finally, ApplyThreshold (Global MCT threshold, Automatic smoothing, 0.3 Threshold correction factor), Morph (skel, once), and MeasureNeurons (Fill small holes, max hole size 50) were used on skeletonized images to obtain neuron measurements and characteristics.

Flow cytometry. For BrdU, pS10-H3, and Sox 2 FACS analysis, mNPCs were washed with cold PBS and fixed in $70 \%$ ethanol at $4{ }^{\circ} \mathrm{C}$ overnight. Cells were pelleted and treated with $2 \mathrm{~N} \mathrm{HCl} /$ Triton X-100 at room temperature for $30 \mathrm{~min}$ to denature DNA. After neutralization, cells were incubated with primary antibody for $30 \mathrm{~min}$ in FACS buffer and washed in PBS. Antibodies anti-BrdU APC (diluted 1:500), anti-pS10-H3-Alexa488 (diluted 1:1000), and anti-Sox2 PE (diluted 1:500) were used. After three washes in cold PBS, cells were resuspended in PBS and analyzed by BD FACSAria ${ }^{\mathrm{Tm}}$ Fusion and FlowJo software.

Neurosphere assay. After filtration of cells derived from embryonic neural tubes, cells were incubated in $0.5 \mu \mathrm{M}$ Neurofluor CDr3 $3^{\text {rw }}+$ DNase I (NEB) for $1 \mathrm{~h}$ in a $37^{\circ} \mathrm{C}$ incubator. CDr3-positive mNPCs were sorted on a BD FACSAria ${ }^{\mathrm{rm}}$ Fusion flow cytometer and plated in ultra-low attachment six-well plates at a density of 10 cells/ $\mu \mathrm{L}$. Neurospheres were allowed to grow for 6 days, with media addition on day 3 and, and samples were imaged on day 6 with a Zeiss AxioObserver D1 at 5x magnification. Neurospheres were collected and treated with Accutase for $5 \mathrm{~min}$ at $37^{\circ} \mathrm{C}$. Cells were counted using trypan blue to assess viability and $10 \mathrm{cells} / \mu \mathrm{l}$ replated in triplicated to derive secondary neurospheres. This process was repeated again after 6 days to derive tertiary neurospheres. Eight images/well were taken for a total of 24 views and analyzed. Image stacks were then created for each cell line or drug treatment set of images. Stacks were created using the FIJI "Images to Stack" menu item (found under the menu "Images $\rightarrow$ Stacks $\rightarrow$ Images to Stack"). A binary images stack was created from the original image stack by using the FIJI plugin "Trainable Weka Segmentation," (found under the FIJI menu "Plugins $\rightarrow$ Segmentation $\rightarrow$ Trainable Weka Segmentation"). Trainable Weka segmentation uses a fast-random forest classification method to create a binary image with neurospheres colored black in the foreground with a blank white background. Binary images stacks were cleaned up using the pencil tool in FIJI, as Trainable Weka Segmentation did not perfectly segment all neurospheres and some needed to be segmented by hand. Clean binary image stacks of neurospheres were then quantified using the "Analyze Particles..." menu item (found under the menu "Analyze $\rightarrow$ Analyze Particles..."). The number of neurospheres and area of each neurosphere were recorded from data generated by the "Analyze Particles" menu item for further data analysis. For the clonal analysis, primary neurospheres were dissociated with Accutase for $5 \mathrm{~min}$ at $37^{\circ} \mathrm{C}$, triturated to single cells, and resuspended in fresh media. After assessing viability and live cell counts using trypan blue, cells were diluted to a concentration of 5 cells $/ 100 \mu \mathrm{l}$ media or 3 cells/ $100 \mu \mathrm{l}$ media for plating in 96 well ultra-low attachment plates. Plates were transferred to incubator and left untouched for 1 week until counting the number of formed neurospheres. For each datapoint, neurospheres were quantified from 20 wells of 5 cells/well or from 30 wells of 3 cells/well.

RNA isolation. Total RNA was isolated from cultured cells and mouse neural tubes (after SOX2-positive FACS sorting from E13.5 embryos) using Ribozol (VWR) and purified using the Direct-zol RNA Purification Kit (Zymo Research). DNA digestion with DNase I was performed as suggested and RNA concentration and purity was measured by NanoDrop (ThermoFisher).

RNA-Seq. Paired-end 100-cycle sequencing was performed on HiSeq 2000 or HiSeq 4000 sequencers, per the manufacturer's directions (Illumina). RNA-seq was mapped and HTSeq (version 0.6.1p1) ${ }^{58}$ was used to estimate fragments per kilobase of transcript per million mapped reads (FPKM) based based on GENCODE $(\mathrm{v} 24)^{59}$. After normalization by trimmed mean of $M$ values (TMM) and filtering out genes not expressed in both groups $($ FPKM $<1)$, Voom was used to identify differentially expressed genes. Volcano plots were generated using ProteinPaint viewer (St. Jude) at cloud.stjude.org hosted by DNANexus. For GSEA analysis ${ }^{60}$, gene sets were put together with MSigDB database ${ }^{61}(\mathrm{C} 2, \mathrm{v} 5.1)$ and analyzed by using prerank mode (version 3.0).

Reverse transcription qPCR. Total RNA (250-1000 ng) was reverse transcribed using the SuperScript IV VILO Master Mix (ThermoFisher). Real-time qPCR reactions were performed with iTaq SYBR ${ }^{\circledR}$ Green Supermix (Bio-Rad 1725124) or PowerUp SYBR ${ }^{\oplus}$ Green Master Mix (ThermoFisher A25778), using Applied Biosystems QuantStudio 3 with primers listed in Supplementary Table S2. For Taqman assays, qPCR reactions were performed with Taqman ${ }^{\boxplus}$ Fast Advanced Master Mix (ThermoFisher 4444964) on the same machine with Taqman assays listed in Supp Table 1. qPCR results were quantified using the difference in threshold cycle values between the gene of interest and the endogenous control by the $2^{-\Delta \Delta \mathrm{Ct}}$ method. All experimental samples were compared to a wild-type littermate or DMSO control.

Wild-type YBX1 and YBX1 mutant construct generation. Amino acids 183-205 in the full-length Ybx1 were deleted to obtain the delNLS mutation. Wild-type human YBX1 was amplified from complementary DNA (cDNA) reverse transcribed from total RNA template of $\mathrm{H} 9$ human embryonic stem cells using primers YBX1-F and YBX1-R (Supplementary Table S3) which contain attB sites for Gateway cloning. The PCR product was used in a BP reaction with pDONR221 and sequence verified by Sanger sequencing. An LR reaction transferred the wildtype YBX1 cDNA to pcDNA-DEST53. All YBX1 mutant constructs were generated by PCR amplification from pDONR221-YBX1 with primers listed in Supplementary Table S3. Fragments were either immediately used in a BP reaction with pDONR221 or for delF2 and delNLS (deletion of amino acids 183-205) - gel extracted, digested with appropriate restriction enzymes and ligated with Quick Ligase (NEB). After sequence verification, constructs were LR cloned into final destination vectors.

Lentiviral construct generation and lentivirus production. To generate lentiviral constructs, YBX1-FL and YBX1-delNLS were excised from pcDNA-DEST53 plasmids via PCR with primers (YBX1(BamHI)-F and YBX1(XhoI)-R). PCR products were double-digested with restriction enzymes in CUTSMART Buffer (NEB). MSCV-IRES-mCHERRY (a gift from Martine Roussel, SJCRH) was also double digested in the same buffer and the backbone fragment isolated by gel extraction. Cut PCR products and backbone were ligated with Quick Ligase and transformed into E.coli TOP10 cells. Plasmids were Sanger sequencing verified before production of lentivirus. To produce lentivirus, viral constructs were cotransfected with pMD Gag-pol and p-VSVg (gifts from Martine Roussel) into HEK 293T cells with Xfect Transfection reagent. Lentiviral particles were harvested into unsupplemented NeuroCult ${ }^{\mathrm{ma}}$ Proliferation Media, and titer quantified by Lenti-X qRT-PCR titration kit (ClonTech). For transduction, Ybx1-KO mNPCs were inoculated with lentiviral particles mixed with fresh proliferation media supplemented with $20 \mathrm{ng} / \mathrm{mL}$ rhEGF. Cells were allowed to recover for two days before FACS sorting for mCHERRY expression. YBX1 expression was confirmed by qRTPCR and immunofluorescence after growth of sorted cells.

Ultra-low input native chromatin immunoprecipitation. Mouse NPCs from Sox2-GFP positive embryos were isolated and sorted. Using an equal number of cells as input and spike-in Drosophila S2 chromatin, nuclei were isolated using Nuclear Isolation Buffer (MilliporeSigma NUC101) and diluted MNase enzyme added to digest DNA. The MNase reaction was carried out for $7.5 \mathrm{~min}$ at $37^{\circ} \mathrm{C}$ and stopped by addition of EDTA. A $1 \%$ Triton/1\% deoxycholate solution was added to lyse nuclei and complete immunoprecipitation buffer added to allow for $\sim 200 \mu \mathrm{l}$ lysate/immunoprecipitation. Antibodies were bound to Protein A/G magnetic beads (ThermoFisher) and after pre-clearing on washed magnetic beads with no 
antibody, antibody-bead complexes were added to chromatin $\mathrm{O} / \mathrm{N}$ at $4{ }^{\circ} \mathrm{C}$. After successive washes in low salt and high salt wash buffers, chromatin was eluted in ChIP elution buffer for $1.5 \mathrm{~h}$ at $65^{\circ} \mathrm{C}$. DNA was extracted using phenolchloroform-isoamyl alcohol extraction and MaXtract phase-lock tubes (QIAGEN) to retain maximal DNA. DNA was further purified using Ampure XP beads (Agencourt) and libraries constructed through the following processes: (1) End repair with T4 PNK, Klenow DNA polymerase, and T4 DNA polymerase; (2) Atailing with dATP and Klenow ( $3^{\prime}-5^{\prime}$ exo-) polymerase; (3) Adapter ligation with annealed Illumina adapters and Quick DNA ligase; and (4) Library amplification with Illumina paired-end indexed primers and 2X Phusion HF Master Mix. Library amplification was performed for 12 cycles and DNA purified by Ampure XP beads and library yield and quality evaluated using a D1000 High Sensitivity screentape on an Agilent TapeStation.

CUT\&RUN. We followed CUT\&RUN described in Skene and Henikoff ${ }^{42}$, with minor variations. Briefly, mNPCs from Sox2-eGFP-positive embryos were isolated and sorted as described above. NPCs and spike-in S2 cells were pelleted and resuspended in wash buffer. Bio-Mag Plus Concanavalin-A coated beads (Bangs Laboratories BP531) were added to cells (diluted in binding buffer) to bind nuclei to beads. Supernatant was removed and samples were blocked for $5 \mathrm{~min}$ at RT with digitonin block buffer. Antibody diluted in digitonin block buffer was added and samples were rotated for $3 \mathrm{~h}, 5 \mathrm{~h}$, or $\mathrm{O} / \mathrm{N}$ in the cold. Beads were collected and washed $3 \times$ with digitonin block buffer before adding pA-MNase to beads. After a 1 - $h$ incubation, beads were washed $3 \times$ and resuspended in wash buffer. After equilibration on ice, $100 \mathrm{mM} \mathrm{CaCl}_{2}$ was added to tubes and samples were incubated for $25 \mathrm{~min}$ with agitation at the 15 -min mark. The reaction was stopped by adding the stop buffer. Samples were incubated at $37^{\circ} \mathrm{C}$ for $30 \mathrm{~min}$ to release chromatin. DNA was isolated by phenol-chloroform-isoamyl alcohol extraction and MaXtract phase-lock tubes (QIAGEN) to maximally retain chromatin. Chromatin was resuspended in low EDTA TE buffer and analyzed by TapeStation using the HS DNA Kit (Agilent). Libraries were made using the Accel-NGS ${ }^{\oplus}$ is Plus DNA Library Kit (Swift Biosciences) and submitted for sequencing. In all cases, $\sim 15 \%$ input was removed and IgG was used as a negative control.

\section{Deep sequencing analysis of chromatin immunoprecipitation or CUT\&RUN} $50 \mathrm{bp}$ single-end reads for ChIP-seq or $50 \mathrm{bp}$ paired-end reads for CUT\&RUN-seq were obtained and aligned to mouse genome assembly $\mathrm{mm} 10$ and fruit fly genome assembly dm6 by BWA (version 0.7.12, default parameter). Duplicated reads were then marked by Picard (version 1.65 [1160]). For ChIP-seq, uniquely mapped reads were retained by SAMtools (parameter "-q $1-\mathrm{F} 1024$ ", version 1.4), and quality control was ensured by following ENCODE criteria ${ }^{62}$. We extended reads to fragment size estimated by SPP63 and then normalized to dm6 sequencing reads number(fruit fly S2 cells). Peaks were inspected on $\mathrm{IGV}^{64}$ and peaks for each replicate were called by MACS2 (version 2.0.9 20111102 option "nomodel" with "extsize" defined as fragment size estimated by SPP) ${ }^{65}$ and SICER (redundancy threshold 1, window size $200 \mathrm{bp}$, effective genome fraction 0.86 , gap size $600 \mathrm{bp}$, FDR 0.00001 with estimated fragment size defined by SPP) ${ }^{66}$ and then merged by bedtools (version 2.17.0) after removing a SICER-called peak if it overlaps MACS2 peaks. For Cut\&Run, properly paired uniquely mapped reads were retained by SAMtools (parameter "-q 1 -F 1804", version 1.4), sorted by name and converted to bedpe format by bedtools. Only fragments shorter than 2000 bp were kept to peak calling and generating of bigwig tracks. Similarly, MACS2 (bedpe mode) and SICER were used for peak calling. To find reproducible peaks, we first called peaks for each replicate twice with an FDR corrected p-value cutoff of 0.05 as a highconfidence peak and an FDR-corrected $P$ value cutoff 0.5 as low-confidence peak set. We only considered high-confidence peaks that also overlap at least lowconfidence peaks in other replicates as reproducible peaks. We did the same by employing SEACR ${ }^{67}$, using the top 1 percentile as the high-confidence peak and the top 5 percentile as the low-confidence peak ("non stringent" mode) and further require the reproducible peaks overlap SEACR reproducible peaks. deepTools ${ }^{68}$ was used to plot the heatmap. Peak overlap was tested by the hypergeometric test with assumed binomial distributions. Gene ontology analysis was generated by Enrichr ${ }^{44}$. For histone modifications, the quality control standard was set as before $^{69}$. Voom ${ }^{70}$ was used to test significant differences between control and Ybx1-KO after TMM normalization. For enhancer analysis, we used active and poised enhancer signature lists from the embryonic brain at E14.5 that were downloaded from the ENCODE portal (https://www.encodeproject.org/) with the identifiers ENCFF682UGG, ENCFF386QNM, and ENCFF998FUV.

Drosophila S2 transfection and protein pull down. Drosophila S2 cells were grown and passaged in Schneider's Drosophila medium (ThermoFisher Scientific) plus $10 \%$ heat-inactivated fetal bovine serum (FBS). For transfections, $1 \times 10^{6}$ cells/ well were seeded in $1 \mathrm{~mL}$ complete medium in six-well plates. Before transfection, medium was removed and replaced with FBS-free Schneider's Drosophila medium. pAMW plasmids were transfected with Cellfectin ${ }^{\text {mot }}$ II Reagent (ThermoFisher) and Opti-MEM medium (ThermoFisher) according to manufacturer's instructions with $6 \mu \mathrm{g}$ plasmid/well. After $72 \mathrm{~h}$, cells were collected by scraping and lysed in Buffer D + PI + DTT. Full-length open reading frame of JARID2 was cloned into pFastBac, using the Bac-to-Bac N-His TOPO cloning kit (Life Technologies) for baculovirus generation via Bac-to-Bac baculovirus expression from the St. Jude Protein Production Facility. Full-length recombinant protein was bound overnight at $4{ }^{\circ} \mathrm{C}$ to Pierce $^{\text {tw }}$ Protein A/G Magnetic Beads (ThermoFisher Scientific) with JARID2 antibody (Novus Biologicals). Protein-bead complexes (or beads with no antibody) were washed with PBST, added to recombinant protein fragment lysate for $4 \mathrm{~h}$ at $4^{\circ} \mathrm{C}$ in HEPM, washed in PBST, and eluted with $0.1 \mathrm{M}$ glycine ( $\mathrm{pH}$ 2.3). Eluates were neutralized with $1.5 \mathrm{M}$ Tris buffer, $\mathrm{pH} 8.8$, and analyzed by Western blotting

Ezh2 inhibitor administration. The Ezh2 inhibitor GSK126 (Cayman Chemical) was dissolved in DMSO to a stock concentration of $\sim 9.5 \mathrm{mM}$. It was further diluted in unsupplemented Neurocult ${ }^{\text {tx }}$ Basal Medium before final dilution (to 100 or $500 \mathrm{nM}$ ) in either Proliferation Media or Differentiation Media, as indicated in figure legends. For the H3K27me3 western blot, mNPCs were treated for $48 \mathrm{~h}$ with drugs at indicated concentrations before harvesting. For NPC qPCR analysis, cells were treated for 6 days with drug, and for differentiation experiments cells were pretreated for 6 days and treated throughout the differentiation course. Media with freshly diluted and the drug was added every 3 days.

Statistics and reproducibility. Statistical parameters including the definitions and exact value of $n$ (e.g., total number of experiments, replications, axons, organelles, or neurons), deviations, $P$ values, and the types of the statistical tests are reported in the figures and corresponding figure legends. Statistical analysis was carried out using Prism 8 (GraphPad Software $)^{27}$. Statistical analysis was conducted on data from three or more biologically independent experimental replicates. Comparisons between groups were planned before statistical testing and target effect sizes were not predetermined. Error bars displayed on graphs represent the mean \pm SEM of at least three independent experiments. All western immunoblots were repeated at least two times with different biological samples. Micrographs are representative of a minimum of four images taken from at least two biological replicates.

Reporting summary. Further information on research design is available in the Nature Research Life Sciences Reporting Summary linked to this article.

\section{Data availability}

All sequencing data are deposited in NCBI GEO database under accession number GSE137853. Mass spectrometry data were deposited in ProteomXchange, with project accession: PXD015670. All other relevant data supporting the key findings of this study are available within the article and its Supplementary Information files or from the corresponding author upon reasonable request. The source data underlying Figs. 1c, d, $\mathrm{f}-\mathrm{h}, \mathrm{k}, 2 \mathrm{~g}, 3 \mathrm{c}-\mathrm{h}, 6 \mathrm{a}-\mathrm{f}, \mathrm{h}$, and $7 \mathrm{~b}-\mathrm{d}, \mathrm{g}-\mathrm{m}, 8 \mathrm{a}, \mathrm{b}, \mathrm{d}-\mathrm{i}$ and Supplementary Figs. S1a-c, S2a, d, e, g, I, j, l, S3c, d, S5i, l, S6e, f, S7a, c, e-g, S8a-h, and S9b-e are provided as a Source Data file. A reporting summary for this article is available as a Supplementary Information file. Source data are provided with this paper.

\section{Code availability}

Code is deposited at https://doi.org/10.6084/m9.figshare.7411835. Source data are provided with this paper.

Received: 3 September 2019; Accepted: 22 July 2020;

Published online: 13 August 2020

\section{References}

1. Gage, F. H. \& Temple, S. Neural stem cells: generating and regenerating the brain. Neuron 80, 588-601 (2013).

2. Kohwi, M. \& Doe, C. Q. Temporal fate specification and neural progenitor competence during development. Nat. Rev. Neurosci. 14, 823-838 (2013).

3. Touma, J. J., Weckerle, F. F. \& Cleary, M. D. Drosophila Polycomb complexes restrict neuroblast competence to generate motoneurons. Development 139, 657-666 (2012).

4. Hirabayashi, Y. et al. Polycomb limits the neurogenic competence of neural precursor cells to promote astrogenic fate transition. Neuron 63, 600-613 (2009).

5. Margueron, R. \& Reinberg, D. The Polycomb complex PRC2 and its mark in life. Nature 469, 343-349 (2011).

6. Simon, J. A. \& Kingston, R. E. Occupying chromatin: Polycomb mechanisms for getting to genomic targets, stopping transcriptional traffic, and staying put. Mol. Cell 49, 808-824 (2013).

7. Tatton-Brown, K. et al. Germline mutations in the oncogene EZH2 cause Weaver syndrome and increased human height. Vol. 2 (2011).

8. Cooney, E. et al. mutation in patient with Weaver syndrome. Am. J. Med. Genet. A 173, 541-545 (2017).

9. Erhardt, S. et al. Consequences of the depletion of zygotic and embryonic enhancer of zeste 2 during preimplantation mouse development. Development 130, 4235-4248 (2003). 
10. Pasini, D., Bracken, A. P., Jensen, M. R., Lazzerini Denchi, E. \& Helin, K. Suz12 is essential for mouse development and for EZH2 histone methyltransferase activity. EMBO J. 23, 4061-4071 (2004).

11. Mager, J., Montgomery, N. D., de Villena, F. P. \& Magnuson, T. Genome imprinting regulated by the mouse Polycomb group protein Eed. Nat. Genet. 33, 502-507 (2003).

12. Shan, Y. et al. PRC2 specifies ectoderm lineages and maintains pluripotency in primed but not naive ESCs. Nat. Commun. 8, 672 (2017).

13. Shan, Y. et al. PRC2 specifies ectoderm lineages and maintains pluripotency in primed but not naïve ESCs. Nat. Commun. 8, 672 (2017).

14. Pasini, D., Bracken, A. P., Hansen, J. B., Capillo, M. \& Helin, K. The polycomb group protein Suz12 is required for embryonic stem cell differentiation. Mol. Cell Biol. 27, 3769-3779 (2007).

15. Takeuchi, T. et al. Gene trap capture of a novel mouse gene, jumonji, required for neural tube formation. Genes Dev. 9, 1211-1222 (1995).

16. Miro, X. et al. Haploinsufficiency of the murine polycomb gene Suz12 results in diverse malformations of the brain and neural tube. Dis. Model Mech. 2, 412-418 (2009).

17. Volcik, K. A. et al. Evaluation of the jumonji gene and risk for spina bifida and congenital heart defects. Am. J. Med. Genet. A 126A, 215-217 (2004).

18. Beringer, M. et al. EPOP functionally links elongin and polycomb in pluripotent stem Cells. Mol. Cell 64, 645-658 (2016).

19. Conway, E. et al. A family of vertebrate-specific polycombs encoded by the LCOR/LCORL genes balance PRC2 subtype activities. Mol. Cell 70, 408-421 (2018). e408.

20. Liefke, R., Karwacki-Neisius, V. \& Shi, Y. EPOP interacts with elongin BC and USP7 to modulate the chromatin landscape. Mol. Cell 64, 659-672 (2016).

21. Deevy, O. \& Bracken, A. P. PRC2 functions in development and congenita disorders. Development 146, https://doi.org/10.1242/dev.181354 (2019).

22. Eliseeva, I. A., Kim, E. R., Guryanov, S. G., Ovchinnikov, L. P. \& Lyabin, D. N. Y-box-binding protein 1 (YB-1) and its functions. Biochem. 76, 1402-1433 (2011).

23. Lasham, A., Print, C. G., Woolley, A. G., Dunn, S. E. \& Braithwaite, A. W. YB1: oncoprotein, prognostic marker and therapeutic target? Biochem J. 449, 11-23 (2013).

24. Kuwano, M., Shibata, T., Watari, K. \& Ono, M. Oncogenic Y-box binding protein-1 as an effective therapeutic target in drug-resistant cancer. Cancer Sci. 110, 1536-1543 (2019).

25. Fotovati, A. et al. YB-1 bridges neural stem cells and brain tumor-initiating cells via its roles in differentiation and cell growth. Cancer Res. 71, 5569-5578 (2011).

26. Dey, A. et al. YB-1 is elevated in medulloblastoma and drives proliferation in Sonic hedgehog-dependent cerebellar granule neuron progenitor cells and medulloblastoma cells. Oncogene 35, 4256-4268 (2016).

27. Lu, Z. H., Books, J. T. \& Ley, T. J. YB-1 is important for late-stage embryonic development, optimal cellular stress responses, and the prevention of premature senescence. Mol. Cell Biol. 25, 4625-4637 (2005).

28. Bhullar, J. \& Sollars, V. E. YBX1 expression and function in early hematopoiesis and leukemic cells. Immunogenetics 63, 337-350 (2011)

29. Uchiumi, T. et al. YB-1 is important for an early stage embryonic development: neural tube formation and cell proliferation. J. Biol. Chem. 281, 40440-40449 (2006)

30. Oliviero, G. et al. Dynamic protein interactions of the polycomb repressive complex 2 during differentiation of pluripotent cells. Mol. Cell Proteom. 15, 3450-3460 (2016).

31. Yun, S. W. et al. Neural stem cell specific fluorescent chemical probe binding to FABP7. Proc. Natl Acad. Sci. USA 109, 10214-10217 (2012).

32. Basaki, Y. et al. Akt-dependent nuclear localization of Y-box-binding protein 1 in acquisition of malignant characteristics by human ovarian cancer cells. Oncogene 26, 2736-2746 (2007).

33. Shanmugalingam, S. et al. Ace/Fgf8 is required for forebrain commissure formation and patterning of the telencephalon. Development 127, 2549-2561 (2000).

34. Carl, M., Loosli, F. \& Wittbrodt, J. Six3 inactivation reveals its essential role for the formation and patterning of the vertebrate eye. Development 129, 4057-4063 (2002).

35. Lagutin, O. V. et al. Six3 repression of Wnt signaling in the anterior neuroectoderm is essential for vertebrate forebrain development. Genes Dev. 17, 368-379 (2003).

36. Yoshida, M. et al. Emx1 and Emx2 functions in development of dorsal telencephalon. Development 124, 101-111 (1997).

37. Kitamura, K. et al. Mutation of ARX causes abnormal development of forebrain and testes in mice and X-linked lissencephaly with abnormal genitalia in humans. Nat. Genet. 32, 359-369 (2002).

38. Bolder, N. M., Wagenaar, J. A., Putirulan, F. F., Veldman, K. T. \& Sommer, M. The effect of flavophospholipol (Flavomycin) and salinomycin sodium (Sacox) on the excretion of Clostridium perfringens, Salmonella enteritidis, and
Campylobacter jejuni in broilers after experimental infection. Poult. Sci. 78, 1681-1689 (1999).

39. Ohtsuka, T., Sakamoto, M., Guillemot, F. \& Kageyama, R. Roles of the basic helix-loop-helix genes Hes1 and Hes5 in expansion of neural stem cells of the developing brain. J. Biol. Chem. 276, 30467-30474 (2001).

40. Hatakeyama, J. et al. Hes genes regulate size, shape and histogenesis of the nervous system by control of the timing of neural stem cell differentiation. Development 131, 5539-5550 (2004).

41. Shimizu, T. et al. Zinc finger genes Fezf1 and Fezf2 control neuronal differentiation by repressing Hes5 expression in the forebrain. Development 137, 1875-1885 (2010).

42. Skene, P. J. \& Henikoff, S. An efficient targeted nuclease strategy for high resolution mapping of DNA binding sites. Elife 6, https://doi.org/10.7554/ eLife.21856 (2017).

43. Brind'Amour, J. et al. An ultra-low-input native ChIP-seq protocol for genome-wide profiling of rare cell populations. Nat. Commun. 6, 6033 (2015).

44. Kuleshov, M. V. et al. Enrichr: a comprehensive gene set enrichment analysis web server 2016 update. Nucleic Acids Res. 44, W90-W97 (2016).

45. McCabe, M. T. et al. EZH2 inhibition as a therapeutic strategy for lymphoma with EZH2-activating mutations. Nature 492, 108-112 (2012).

46. Fan, T. et al. EZH2-dependent suppression of a cellular senescence phenotype in melanoma cells by inhibition of p21/CDKN1A expression. Mol. Cancer Res. 9, 418-429 (2011)

47. Pereira, J. D. et al. Ezh2, the histone methyltransferase of PRC2, regulates the balance between self-renewal and differentiation in the cerebral cortex. Proc. Natl Acad. Sci. USA 107, 15957-15962 (2010).

48. Zhao, L. et al. Ezh2 is involved in radial neuronal migration through regulating Reelin expression in cerebral cortex. Sci. Rep. 5, 15484 (2015)

49. Tronche, F. et al. Disruption of the glucocorticoid receptor gene in the nervous system results in reduced anxiety. Nat. Genet. 23, 99-103 (1999).

50. $\mathrm{Yu}, \mathrm{M}$. et al. Insights into GATA-1-mediated gene activation versus repression via genome-wide chromatin occupancy analysis. Mol. Cell 36, 682-695 (2009).

51. Zemke, M. et al. Loss of Ezh2 promotes a midbrain-to-forebrain identity switch by direct gene derepression and Wnt-dependent regulation. BMC Biol. 13, 103 (2015).

52. Comet, I., Riising, E. M., Leblanc, B. \& Helin, K. Maintaining cell identity: PRC2-mediated regulation of transcription and cancer. Nat. Rev. Cancer 16, 803-810 (2016).

53. Chattopadhyay, R. et al. Regulatory role of human AP-endonuclease (APE1/ Ref-1) in YB-1-mediated activation of the multidrug resistance gene MDR1. Mol. Cell Biol. 28, 7066-7080 (2008).

54. Pombero, A., Valdes, L., Vieira, C. \& Martinez, S. Developmental mechanisms and experimental models to understand forebrain malformative diseases. Genes Brain Behav. 6(Suppl 1), 45-52 (2007).

55. Eng, J. K., McCormack, A. L. \& Yates, J. R. An approach to correlate tandem mass spectral data of peptides with amino acid sequences in a protein database. J. Am. Soc. Mass Spectrom. 5, 976-989 (1994).

56. Zhou, J. Y. et al. Galectin-3 is a candidate biomarker for amyotrophic lateral sclerosis: discovery by a proteomics approach. J. Proteome Res. 9, 5133-5141 (2010).

57. Arnold, K. et al. Sox $2(+)$ adult stem and progenitor cells are important for tissue regeneration and survival of mice. Cell Stem Cell 9, 317-329 (2011).

58. Anders, S., Pyl, P. T. \& Huber, W. HTSeq-a Python framework to work with high-throughput sequencing data. Bioinformatics 31, 166-169 (2015).

59. Harrow, J. et al. GENCODE: the reference human genome annotation for The ENCODE Project. Genome Res. 22, 1760-1774 (2012).

60. Subramanian, A. et al. Gene set enrichment analysis: a knowledge-based approach for interpreting genome-wide expression profiles. Proc. Natl Acad. Sci. 102, 15545-15550 (2005).

61. Liberzon, A. et al. The molecular signatures database hallmark gene set collection. Cell Syst. 1, 417-425 (2015).

62. Landt, S. G. et al. ChIP-seq guidelines and practices of the ENCODE and modENCODE consortia. Genome Res. 22, 1813-1831 (2012).

63. Kharchenko, P. V., Tolstorukov, M. Y. \& Park, P. J. Design and analysis of ChIP-seq experiments for DNA-binding proteins. Nat. Biotechnol. 26, 1351-1359 (2008)

64. Robinson, J. T. et al. Integrative genomics viewer. Nat. Biotechnol. 29, 24-26 (2011).

65. Zhang, Y., Shin, H., Song, J. S., Lei, Y. \& Liu, X. S. Identifying positioned nucleosomes with epigenetic marks in human from ChIP-Seq. BMC Genom. 9, 537 (2008)

66. Zang, C. et al. A clustering approach for identification of enriched domains from histone modification ChIP-Seq data. Bioinformatics 25, 1952-1958 (2009).

67. Meers, M. P., Tenenbaum, D. \& Henikoff, S. Peak calling by Sparse Enrichment Analysis for CUT\&RUN chromatin profiling. Epigenetics Chromatin 12, 42 (2019). 
68. Ramírez, F. et al. deepTools2: a next generation web server for deepsequencing data analysis. Nucleic Acids Res. 44, W160-W165 (2016).

69. Aldiri, I. et al. The dynamic epigenetic landscape of the retina during development, reprogramming, and tumorigenesis. Neuron 94, 550-568. e510 (2017).

70. Law, C. W., Chen, Y., Shi, W. \& Smyth, G. K. voom: precision weights unlock linear model analysis tools for RNA-seq read counts. Genome Biol. 15, R29 (2014).

\section{Acknowledgements}

The authors thank A. Andersen and M.F. Roussel for discussions, T.J. Ley for the Ybx1KO mouse strain; A. Andersen and V. Shanker for editing the manuscript; J. Houston for FACS; S. Olsen and D. Roeber for sequencing samples; L. Ding, M. J. Robert, and M. Rusch for sequencing mapping; M. Sahnine, J. Klein, I. Lam, H. Chen, A. Dash, and K. Kleinrichert for experimental assistance. Images were acquired at the Cell \& Tissue Imaging Center, which is supported by SJCRH and NCI P30 (CA021765). M.E. is funded by NIH (1F32HD093276). B.X. and Y.F. are supported by NCI P30 (CA21765). This research is funded by American Lebanese Syrian Associated Charities, American Cancer Society (132096-RSG-18-032-01-DDC), and NIH (1R01GM134358-01). The content is solely the responsibility of the authors and does not necessarily represent the official views of the National Institutes of Health.

\section{Author contributions}

M.E.: most experiments and analyzed data. Y.M.: genetic crosses, NPC FACS, MEF studies, CUT\&RUN and library prep, and histone WB. B.X.: sequencing analyses. C.W.: co-IP followed by mass spectrometry or WB. J.L.: genetic crosses and neurosphere assay. L.M.: cryosection and image quantification. Y.F.: supervision of B.X. V.P.: mass spectrometry analysis. J.C.P.: designed the project, analyzed data, and wrote the manuscript with inputs from all authors.

\section{Competing interests}

The authors declare no competing interests.

\section{Additional information}

Supplementary information is available for this paper at https://doi.org/10.1038/s41467020-17878-y.

Correspondence and requests for materials should be addressed to J.C.P.

Peer review information Nature Communications thanks Haruhiko Koseki, Michiel Vermeulen and the other, anonymous, reviewer(s) for their contribution to the peer review of this work. Peer reviewer reports are available.

Reprints and permission information is available at http://www.nature.com/reprints

Publisher's note Springer Nature remains neutral with regard to jurisdictional claims in published maps and institutional affiliations.

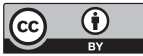

Open Access This article is licensed under a Creative Commons Attribution 4.0 International License, which permits use, sharing, adaptation, distribution and reproduction in any medium or format, as long as you give appropriate credit to the original author(s) and the source, provide a link to the Creative Commons license, and indicate if changes were made. The images or other third party material in this article are included in the article's Creative Commons license, unless indicated otherwise in a credit line to the material. If material is not included in the article's Creative Commons license and your intended use is not permitted by statutory regulation or exceeds the permitted use, you will need to obtain permission directly from the copyright holder. To view a copy of this license, visit http://creativecommons.org/licenses/by/4.0/.

(C) The Author(s) 2020, corrected publication 2023 